Geometry $\&$ Topology

Volume 8 (2004) 475-509

Published: 16 February 2004

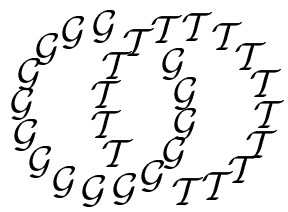

\title{
Permutations, isotropy and smooth cyclic group actions on definite 4 -manifolds
}

\author{
IAN HAMBLETON \\ Mihail TANASE \\ Department of Mathematics and Statistics \\ McMaster University, Hamilton, ON L8S 4K1, Canada \\ Email: ian@math.mcmaster.ca and tanasem@math.mcmaster.ca
}

\begin{abstract}
We use the equivariant Yang-Mills moduli space to investigate the relation between the singular set, isotropy representations at fixed points, and permutation modules realized by the induced action on homology for smooth group actions on certain 4-manifolds.
\end{abstract}

AMS Classification numbers Primary: 58D19, 57S17

Secondary: $70 \mathrm{~S} 15$

Keywords: Gauge theory, 4-manifolds, group actions, Yang-Mills, moduli space

Proposed: Ronald Fintushel

Seconded: Ronald Stern, Robion Kirby
Received: 29 July 2003

Revised: 17 January 2004 


\section{Introduction}

We are interested in a kind of "rigidity" for finite group actions on certain smooth 4-manifolds, namely those constructed by connected sums of geometric pieces such as algebraic surfaces. We can ask how closely a smooth, orientationpreserving, finite group action on such a connected sum resembles a equivariant connected sum of algebraic actions on the individual factors.

Following 12, we consider this question for the simplest case, where

$$
X=\#_{1}^{n} \mathbb{C} P^{2}=\mathbb{C} P^{2} \# \ldots \# \mathbb{C} P^{2}
$$

is the connected sum of $n$ copies of the complex projective plane. In that paper we restricted ourselves to actions which induced the identity on the homology of $X$, but here we remove this assumption. Since the intersection form of $X$ is the standard definite form $Q_{X}=\langle 1\rangle \perp \cdots \perp\langle 1\rangle$, we note that its automorphism group is given by an extension

$$
1 \rightarrow\{ \pm 1\}^{n} \rightarrow \operatorname{Aut}\left(H_{2}(X ; \mathbf{Z}), Q_{X}\right) \rightarrow \Sigma_{n} \rightarrow 1
$$

where $\Sigma_{n}$ denotes the group of permutations of $n$ elements. Therefore, if a finite group $\pi$ of odd order acts smoothly on $X$, then $H_{2}(X ; \mathbf{Z})$ is the direct sum of permutation modules of the form $\mathbf{Z}\left[\pi / \pi_{\alpha}\right]$, for various stabilizer subgroups $\pi_{\alpha} \subseteq \pi$.

Remark Our results (like those of [12]) actually hold for any smooth, closed, simply-connected 4-manifold with positive definite intersection form. This is basically due to the result of S Donaldson [3], that any such 4-manifold $X$ has the standard positive definite intersection form, and hence is homotopy equivalent to $\#_{1}^{n} \mathbb{C} P^{2}$ for some $n \geq 0$. With this information, it is easy to see that the arguments go through without essential changes.

We would like to understand how the following three invariants of such an action $(X, \pi)$ are related:

(A) The permutation representation of $\pi$ on $H_{2}(X ; \mathbf{Z})$.

(B) The singular set of the action, meaning the collection of isotropy subgroups and fixed sets $\operatorname{Fix}\left(X, \pi^{\prime}\right)$ for $\pi^{\prime} \subset \pi$.

(C) The tangential isotropy representations $\left(T_{x} X, \pi_{x}\right)$ at all singular points $x \in X$. 
In the rest of the paper, we assume that $\pi=C_{m}$ is a finite cyclic group of odd order $m$, acting smoothly on $X=\#_{1}^{n} \mathbb{C} P^{2}$. We obtain many examples of such smooth actions by starting with linear actions of $\pi$ on $\mathbb{C} P^{2}$. These are just the cyclic subgroups of the algebraic automorphism group $P G L_{3}(\mathbb{C})$, given by sending a generator

$$
t \mapsto\left(\begin{array}{ccc}
1 & & \\
& \zeta^{a} & \\
& & \zeta^{b}
\end{array}\right)
$$

where $\zeta=e^{\frac{2 \pi i}{m}}$ is a primitive $m^{t h}$ root of unity, and $a, b$ are integers such that the greatest common divisor $(a, b, m)=1$. In this case, $\pi$ acts by the identity on homology, and the singular set always contains the three fixed points $[1,0,0],[0,1,0]$, and $[0,0,1]$. In addition, there can be up to three invariant 2 -spheres with various isotropy subgroups, depending on the values of $a$ and $b$. For example, if $(a, b)=(10,3)$ and $m=105$, then the action has 5 orbit types (the maximal number for $\mathbb{C} P^{2}$ ). The tangential representations at the three $\pi$-fixed points are given by the rotation numbers $(a, b),(-a, b-a)$ and $(a-b,-b)$ standing for the decomposition of $T_{x} \mathbb{C} P^{2}=\mathbb{R}^{4}=\mathbb{C}^{2}$ into eigenspaces under the action of $t$. These rotation numbers are well-defined modulo $m$ up to identifying $(a, b) \equiv(b, a) \equiv(-a,-b)$.

Examples of smooth $\pi$-actions on a connected sum $X=\#_{1}^{n} \mathbb{C} P^{2}$ with more isotropy groups and various permutation actions on homology are constructed by a tree of equivariant connected sums, where we connect either at fixed points of two linear actions or along an orbit of singular points. In order to preserve orientation, the rotation numbers at the attaching points must be of the form $(a, b)$ and $(a,-b)$. By this means, we can obtain a large supply of model actions, for which one should be able to work out the relation between (A), (B) and (C).

What can one say about a general smooth action $(X, \pi)$ ? It is not hard to verify that the singular set consists of a configuration of isolated points and 2 -spheres, as in the linear models (see [6]). The main result of [16] generalizes [12, Theorem C]:

Theorem A Let $X$ be a smooth, closed, simply-connected 4-manifold with a positive definite intersection form. Let $\pi$ be a cyclic group of odd order, acting smoothly on on $X$. Then there exists an equivariant connected sum of linear actions on $\mathbb{C} P^{2}$ with the same isotropy structure, singular set and rotation numbers, and the same permutation action on $H_{2}(X ; \mathbf{Z})$ as for the given action $(X, \pi)$. 
This result is proved by using the symmetries of the equivariant Yang-Mills moduli space [1] to produce a stratified equivariant cobordism between $(X, \pi)$ and a connected sum of linear actions, relating the invariants (A), (B) and (C). The following result of [16] holds for smooth, but not for topological actions, by an example of A Edmonds [7.

Theorem B Let $(X, \pi)$ be a smooth action as above, with discrete singular set. Then the action $(X, \pi)$ is semi-free.

In principle, it should now be possible to say exactly which permutation modules can be realized by smooth actions on $\#_{1}^{n} \mathbb{C} P^{2}$ just by studying the equivariant connected sums. We will show that not all modules are realizable: for example if $\pi=C_{p^{k}}$ for $k \geq 3$, then the module $\mathbf{Z}\left[C_{p}\right] \oplus \mathbf{Z}\left[C_{p^{i}}\right], 2 \leq i<k$, is not realizable by any smooth $\pi$-action on $\#_{1}^{n} \mathbb{C} P^{2}$. However, we have the following "stable" realization result. We say that two subgroups $\pi_{1}$ and $\pi_{2}$ of $\pi$ are disjoint if $\pi_{1} \cap \pi_{2}=\{1\}$.

Theorem $\mathbf{C}$ Let $\mathcal{S}$ denote a set of subgroups of $\pi=C_{m}$ containing at most two maximal elements (under inclusion of subgroups). If $\mathcal{S}$ has two maximal elements, suppose that they are disjoint. Then there exists an integer $N=$ $N(\mathcal{S}) \leq m$ such that any permutation module

$$
\bigoplus\left\{\mathbf{Z}\left[\pi / \pi_{\alpha}\right]^{k_{\alpha}}: \pi_{\alpha} \in \mathcal{S}\right\}
$$

is realizable by a smooth $\pi$-action on some $\#_{1}^{n} \mathbb{C} P^{2}$, provided that the multiplicity $k_{\alpha}>N$ for each of the maximal stabilizer subgroups.

Acknowledgement Our interest in permutation actions on homology was stimulated by a talk of A Edmonds [7] at the CRM, Montréal (August 2000) on the orbit types of locally linear topological actions on positive definite 4manifolds. This research was partially supported by NSERC Discovery Grant A4000. The first author also wishes to thank the SFB 478, Universität Münster, for hospitality and support.

\section{The linear models}

We investigate the orbit structure and possible permutation actions that can arise on an equivariant connected sum of linear $\pi$-actions on $\mathbb{C} P^{2}$. Let $(X, \pi)$ denote such a connected sum (always assuming that $X$ is simply-connected), and fix a generator $t$ for the cyclic group $\pi=C_{m}$ of odd order $m$. 


\section{(1.A) Linear actions on $\mathbb{C} P^{2}$}

We have already pointed out that a linear $\pi$-action on $\mathbb{C} P^{2}$ (in standard form) has three fixed points $p_{1}=[1,0,0], p_{2}=[0,1,0]$, and $p_{3}=[0,0,1]$. At these points, the tangential isotropy representations are described by the rotation numbers $(a, b),(-a, b-a)$ and $(-b, a-b)$, considered as pairs of integers mod $m$. For example, $(a, b)$ stands for the linear $\pi$ representation $\mathbb{C}\left(\zeta^{a}\right) \oplus \mathbb{C}\left(\zeta^{b}\right)$. The condition for an effective action of $\pi$ on $\mathbb{C} P^{2}$ is just that the greatest common divisor $(a, b, m)=1$. Up to oriented equivalence, we can't distinguish the representations $(a, b)$ from $(b, a)$ or $(-a,-b)$ so these pairs of rotation numbers are all identified. It will be convenient to use the notation $\mathbb{C} P^{2}(a, b ; m)$ for this linear action, or just $\mathbb{C} P^{2}(a, b)$ if the cyclic group is understood.

Next we observe that the standard linear actions on $\mathbb{C} P^{2}$ always have at least three invariant complex lines (topologically 2-spheres), namely the spans $S_{1}=$ $\left[p_{2}, p_{3}\right], S_{2}=\left[p_{1}, p_{3}\right]$ and $S_{3}=\left[p_{1}, p_{2}\right]$. These invariant 2 -spheres are contained in the fixed sets of the subgroups of order $m_{1}=(a-b, m), m_{2}=(b, m)$ and $m_{3}=(a, m)$ respectively. Notice that the orders $m_{1}, m_{2}$ and $m_{3}$ are all coprime, so that distinct 2 -spheres in the singular set of $\left(\mathbb{C} P^{2}, \pi\right)$ are fixed by distinct subgroups of $\pi$, which intersect only in the identity element. Any three divisors of $m$ can be obtained this way by appropriate choice of the rotation numbers, so we can obtain actions with up to five distinct orbit types. Apart from the fixed points and free orbits, the other three isotropy subgroups may be chosen arbitrarily, subject only to the condition that any two intersect in the identity. In the special case when $m=p^{k}$ for some prime $p$, the divisibility condition implies that there are at most three distinct orbit types. For any $\mathbb{C} P^{2}(a, b)$ the fixed set of each subgroup of $\pi$ consists either of (i) three isolated points $\left(p_{1}, p_{2}\right.$, and $\left.p_{3}\right)$, or (ii) one isolated point and a disjoint 2 -sphere (one of the pairs $\left.p_{i}, S_{i}\right)$.

\section{(1.B) Linear actions on $S^{4}$}

We take $S^{4}$ as the unit sphere in $\mathbb{R}^{5}=\mathbb{R} \oplus \mathbb{C} \oplus \mathbb{C}$ and write the action of our generator in standard diagonal form:

$$
t \mapsto\left(\begin{array}{ccc}
1 & & \\
& \zeta^{a} & \\
& & \zeta^{b}
\end{array}\right)
$$

as a matrix in $S O(5)$. The action is again effective if and only if $(a, b, m)=1$. We will use the notation $S^{4}(a, b ; m)$ for this action. 
For these actions on $S^{4}$, we always have two fixed points $p_{1}=(1,0,0)$ and $p_{2}(-1,0,0)$ with the rotation numbers $(a, b)$ and $(a,-b)$. In addition, the unit 3 -sphere $S^{3} \subset 0 \oplus \mathbb{C} \oplus \mathbb{C}$ always has two $\pi$-invariant circles from the two complex factors, so we get two invariant 2 -spheres in the action. These are $S_{1}=\left\{\left(x, z_{1}, 0\right)\left|x^{2}+\right| z_{1} \mid=1\right\}$ and $S_{2}=\left\{\left(x, 0, z_{2}\right)\left|x^{2}+\right| z_{2} \mid=1\right\}$, which are just the fixed sets of the subgroups of order $m_{1}=(a, m)$ and $m_{2}=(b, m)$. Again these two isotropy subgroups intersect only in the identity, and we can obtain up to four orbit types in general. If $m=p^{k}$ for some prime $p$, then three orbit types is the maximum possible. For any $S^{4}(a, b)$ the fixed set of each subgroup of $\pi$ consists either of (i) two isolated points $\left(p_{1}\right.$ and $p_{2}$ ), or (ii) a 2-sphere (either $S_{1}$ or $S_{2}$ ).

One common feature of these two linear models can be seen in any smooth action on a connected sum.

Theorem 1.1 If a cyclic group $\pi$ of odd order acts smoothly on a closed, smooth simply-connected 4-manifold $X$ with positive definite intersection form, then the fixed point set $\operatorname{Fix}\left(X, \pi^{\prime}\right)$ for any non-trivial subgroup $\pi^{\prime} \subset \pi$ is a union of isolated points and 2-spheres.

Proof Since $X \simeq \#_{1}^{n} \mathbb{C} P^{2}$ for some $n \geq 0$, it follows that there is a standard basis $\left\{e_{1}, \ldots, e_{n}\right\}$ for $H_{2}(X ; \mathbf{Z})$ on which $\pi$ acts by permutations. Let $\pi^{\prime} \neq 1$ be a non-trivial subgroup of $\pi$, and let $C_{p} \subseteq \pi^{\prime}$ be a subgroup of odd prime order $p$. Since the action is orientation-preserving, $\operatorname{Fix}\left(X, C_{p}\right)$ is a disjoint union of isolated points and oriented surfaces. Since $\pi$ and therefore the subgroup $C_{p}$ acts by permutations, the decomposition of $H_{2}(X ; \mathbf{Z})$ as a $\mathbf{Z}\left[C_{p}\right]$-module has no summands of cyclotomic type (see [6, Proposition 1.1] for this terminology). Therefore, by [6. Proposition 2.4] the fixed set has zero first homology, and the result follows.

\section{(1.C) Equivariant connected sums}

To define the equivariant connected sum of two smooth 4-dimensional $\pi$-manifolds $(X, \pi)$ and $(Y, \pi)$, we select fixed points, $x \in X$ and $y \in Y$ with rotation numbers of the form $(a, b)$ on $T_{x} X$ and $(a,-b)$ on $T_{y} Y$. This just means that the tangent representations are equivalent by an orientation-reversing isomorphism. We now construct as usual the connected sum by removing small $\pi$-invariant disks centered at the fixed points, and equivariantly identifying concentric annuli around $x$ and $y$ via the exponential map. Then $X \# Y$ becomes a smooth $\pi$-manifold in the standard way. 
More generally, if $\left(Y, \pi^{\prime}\right)$ is a smooth $\pi^{\prime}$-action, for some subgroup $\pi^{\prime} \subseteq \pi$, we will describe the connected sum of $(X, \pi)$ with $(Z, \pi)=\left(\pi \times_{\pi^{\prime}} Y\right)$ along an orbit of $\pi^{\prime}$-fixed points. Select a point $x \in X_{\left(\pi^{\prime}\right)}:=\left\{x \in X \mid \pi_{x}=\pi^{\prime}\right\}$, and a point $y \in \operatorname{Fix}\left(Y, \pi^{\prime}\right)$ whose rotation numbers $\left(\bmod \left|\pi^{\prime}\right|\right)$ agree with those of $x$ up to orientation-reversal as above. Then we perform the connected sum operation $\pi / \pi^{\prime}$-equivariantly, matching the points of the orbit of $x$ in $X$ with the orbit $\pi / \pi^{\prime} \times\{y\}$. The resulting smooth $\pi$-manifold is denoted $X \#\left(\pi \times \pi_{\pi^{\prime}} Y\right)$.

We will refer to either of these operations as the equivariant connected sum. The first is just a special case of the second when $\pi^{\prime}=\pi$. Notice that when $x \in X$ lies on a 2 -sphere fixed by $\pi^{\prime}$, then the rotation numbers at $x$ have the form $(a, 0) \bmod \left|\pi^{\prime}\right|$. The orientation-reversing matching condition implies that the rotation numbers at $y \in Y$ equal $(-a, 0) \equiv(a, 0) \bmod \left|\pi^{\prime}\right|$, so the point $y$ lies on a fixed 2 -sphere in $Y$.

Since the $\pi^{\prime}$-rotation numbers are constant along connected components of $\pi^{\prime}$ fixed sets, we have some choice in selecting the points at which to perform the connected sum.

Lemma 1.2 The $\pi$-diffeomorphism type of the equivariant connected sum $X \#\left(\pi \times_{\pi^{\prime}} Y\right)$ is independent of the choices of $x$ and $y$ within connected components of $X_{\left(\pi^{\prime}\right)}$ and $\operatorname{Fix}\left(Y, \pi^{\prime}\right)$.

Proof This follows from an equivariant version of the isotopy extension theorem (see [2, VI.3]).

Remark 1.3 In an equivariant connected sum of linear actions on $\mathbb{C} P^{2}$ (or $\left.S^{4}\right)$, the points of $\pi / \pi^{\prime} \times\{x\}$ and $\pi / \pi^{\prime} \times\{y\}$ in the construction described above will be called connecting points. If one connects up copies of $\left(\mathbb{C} P^{2}, \pi\right)$ using only $\pi$-fixed connecting points, the induced action on the homology of the connected sum obtained is trivial. Otherwise there exists at least one nontrivial permutation module $\mathbf{Z}\left[\pi / \pi^{\prime}\right]$ in the homology of the connected sum.

Definition 1.4 Let $\pi^{\prime} \subseteq \pi$ be a subgroup of order $m^{\prime} \mid m$. We say that the linear actions $X=\mathbb{C} P^{2}(a, b ; m)$ and $Y=\mathbb{C} P^{2}\left(a^{\prime}, b^{\prime} ; m^{\prime}\right)$ have a pair of matching $\pi^{\prime}$-fixed components if there exists components $C_{1} \subset X_{\left(\pi^{\prime}\right)}$ and $C_{2} \subset$ $\operatorname{Fix}\left(Y, \pi^{\prime}\right)$ whose rotation numbers on $T_{x} X$ at $x \in C_{1}$ and on $T_{y} Y$ at $y \in C_{2}$ agree $\left(\bmod \left|m^{\prime}\right|\right)$ after an orientation-reversing equivalence. 


\section{(1.D) Trees, roots and branches}

We are going to associate a weighted tree to each equivariant connected sum of linear actions on $\mathbb{C} P^{2}$. Given a positive odd integer $m=|\pi|$ and a positive integer $n$, we will consider $\pi$-equivariant trees $\mathbf{T}$ with $n$ (type $\mathrm{I}$ ), or $n+1$ vertices (type II), having the following properties (see [15, Section 2]):

(i) The vertices and edges of $\mathbf{T}$ are permuted by the $\pi$-action, preserving the incidence relation.

(ii) There is a $\pi$-fixed vertex called the root vertex for the tree. We number the vertices $V=\left\{v_{1}, \ldots, v_{n}\right\}$ (type I) or $V=\left\{v_{0}, v_{1}, \ldots, v_{n}\right\}$ (type II) so that the vertex $v_{1}$ (respectively $v_{0}$ in type II) is the root vertex.

(iii) For a type II tree, the root vertex $v_{0}$ is the unique $\pi$-fixed vertex.

(iv) There is a $\pi$-invariant partial ordering on the vertex set $V\left(v_{i}<v_{j}\right.$ implies $g \cdot v_{i}<g \cdot v_{j}$ for all $g \in \pi$ ), and the root is the unique minimal element in this partial ordering.

(v) The edge set $E$ is $\pi$-invariantly directed so that for each $e \in E$, the initial and terminal vertices $v_{i}=\partial_{0} e$ and $v_{j}=\partial_{1} e$ satisfy $v_{i}<v_{j}$.

We are going to think of the vertices $\left\{v_{1}, \ldots, v_{n}\right\}$ of $\mathbf{T}$ as representing standard linear actions $\mathbb{C} P^{2}\left(a_{i}, b_{i} ; m_{i}\right)$, for $1 \leq i \leq n$. The root vertex $v_{1}$ of a type $\mathrm{I}$ tree will have $m_{1}=m$. For the type II trees, the root vertex $v_{0}$ will be of the form $S^{4}\left(a_{0}, b_{0} ; m_{0}\right)$, with $m_{0}=m$. We will translate from divisors of $m$ to subgroups by letting $\pi_{i}$ denote the subgroup of order $m_{i}$. This discussion should motivate the following definition.

Definition 1.5 An admissible, weighted tree $(\mathbf{T}, \pi)$ is a $\pi$-equivariant, partially-ordered, directed tree with the properties listed above, and in addition:

(i) Each vertex $v_{i}$ has weights $\left(a_{i}, b_{i} ; m_{i}\right)$, where $m_{i}$ is a divisor of $m$, and $a_{i}, b_{i}$ are a pair of integers whose common divisor $\left(a_{i}, b_{i}, m_{i}\right)=1$.

(ii) If $v_{i}<v_{j}$, then $m_{j}$ divides $m_{i}$.

(iii) The weights of $v_{i}$ are the same as those of $g \cdot v_{i}$, for all $g \in \pi$.

(iv) If $v_{i}=\partial_{0} e$ and $v_{j}=\partial_{1} e$ for some edge $e \in E$, then the linear actions $\mathbb{C} P^{2}\left(a_{i}, b_{i} ; m_{i}\right)$ and $\mathbb{C} P^{2}\left(a_{j}, b_{j} ; m_{j}\right)$ have a pair of matching $\pi_{j}$-fixed components

We use the same equivalence relation on the weights as for rotation numbers. In other words, in the triple $\left(a_{i}, b_{i} ; m_{i}\right)$ the pair $\left(a_{i}, b_{i}\right)$ is well-defined only 
$\bmod m_{i}$, and $\left(a_{i}, b_{i}\right)=\left(b_{i}, a_{i}\right)=\left(-a_{i},-b_{i}\right)$. Let $\mathcal{R}$ denote the collection of weights for the vertices of $(\mathbf{T}, \pi)$, and let $\mathbb{T}=(\mathbf{T}, \pi, \mathcal{R})$ denote an admissible weighted tree as defined above. There is an obvious notion of equivalence between weighted trees $\mathbb{T}$ and $\mathbb{T}^{\prime}$, involving a bijection of vertex sets and edge sets which is compatible with the $\pi$-action, incidence relations, and the weights. The ordering structure is useful internally for describing the construction of equivariant connected sums from this data, but one could attach the various $\mathbb{C} P^{2}$ 's is many different orders.

Lemma 1.6 Given an admissible, weighted tree $\mathbb{T}$, the set $\mathbf{T}_{0}$ of $\pi$-fixed vertices and edges is an admissible, weighted subtree $\mathbb{T}_{0} \subseteq \mathbb{T}$.

Proof In particular, we are asserting that $\mathbf{T}_{0}$ is connected, and of course it contains the root vertex by definition. The proof is immediate from the partial ordering property: any $\pi$-fixed vertex $v_{i}$ is connected to the root vertex by a path of edges. It follows that $m_{j}=m$ for any intermediate vertex on the path, so the path lies in $\mathbf{T}_{0}$.

We will call this $\pi$-invariant subtree the homologically trivial subtree of $\mathbb{T}$, since it gives rise to a homologically trivial action. Notice that the complement $\mathbb{T} \backslash \mathbb{T}_{0}$ is a disjoint union of $\pi$-orbits of admissible, weighted trees for various subgroups $\pi^{\prime} \subset \pi$. We call these the branches in $\mathbb{T}_{0}$. For each branch, we can repeat the process to find the homologically trivial $\pi^{\prime}$-subtree of the branch. This gives a canonical way to decompose the tree into simpler pieces.

Theorem 1.7 Given an admissible, weighted tree $\mathbb{T}$, there is an equivariant connected sum of linear actions $(X(\mathbb{T}), \pi)$ such that $X(\mathbb{T})=\#_{1}^{n} \mathbb{C} P^{2}$, up to diffeomorphism. Conversely, given an equivariant connected sum $(X, \pi)$ of linear actions with $X=\#_{1}^{n} \mathbb{C} P^{2}$, there is an admissible, weighted tree $\mathbb{T}$ such that $(X(\mathbb{T}), \pi)=(X, \pi)$. Two trees $\mathbb{T}$ and $\mathbb{T}^{\prime}$ give $\pi$-equivariantly diffeomorphic actions if and only if they are equivalent.

Proof Given an admissible, weighted tree $\mathbb{T}$ we can construct an action on $\#_{1}^{n} \mathbb{C} P^{2}$, using the data given by the weights. We call these $(X(\mathbb{T}), \pi)$ tree manifolds. The last condition on edges allows us to connect the vertex $v_{i}=$ $\mathbb{C} P^{2}\left(a_{i}, b_{i} ; m_{i}\right)$ to $v_{j}=\mathbb{C} P^{2}\left(a_{j}, b_{j} ; m_{j}\right)$ by equivariant connected sum. To see that equivalent trees give rise to equivariantly diffeomorphic actions, we start with the homologically trivial subtree $\mathbb{T}_{0}$. By the "matching" condition on edges, we see that the weights at every vertex in $\mathbb{T}_{0}$ are determined by the 
weights at any single vertex. This is because we are taking the connected sum at $\pi$-fixed points, whose rotation numbers within each $\mathbb{C} P^{2}(a, b ; m)$ are in the set $(a, b),(-a, b-a)$, and $(-b, a-b)$. In forming the equivariant connected sum we pick one of these, say $(a, b)$ and attach $\mathbb{C} P^{2}(a,-b ; m)$. Since $\mathbb{T}_{0}$ is connected, we determine all the rotation numbers in $\mathbb{T}_{0}$ by this process.

Conversely, suppose that $(X, \pi)$ is an equivariant connected sum of actions on $\#_{1}^{n} \mathbb{C} P^{2}$. We will argue by induction on $n$, where the case $n=1$ is clear. We could also encounter the case $n=0$, which is just $S^{4}(a, b ; m)$. By construction, there exists a $\pi$-orbit $\{g \cdot e \mid g \in \pi\}$ in $H_{2}(X ; \mathbf{Z})$, where $e \in H_{2}(X ; \mathbf{Z})$ is represented by some $\mathbb{C} P^{1} \subset \mathbb{C} P^{2}$ used in the equivariant connected sum operation. This factor is $\pi^{\prime}$-invariant, where $\pi^{\prime} \subset \pi$ is the stabilizer of $e$. We may choose $\pi^{\prime}$ a minimal element among the set of isotropy subgroups. Let $\left(X_{0}, \pi\right)$ be the result of removing a $\pi$-orbit of a $\pi^{\prime}$-equivariant tubular neighbourhood $\nu\left(\mathbb{C} P^{1} \subset \mathbb{C} P^{2}\right)$ from $X$, and then gluing in a $\pi$-orbit of $\pi^{\prime}$-invariant 4 -disks. We obtain $\left(X_{0}, \pi\right)$, which by induction is a disjoint union of tree-manifolds and $(Y, \pi)=\pi \times \times_{\pi^{\prime}} \mathbb{C} P^{2}\left(a^{\prime}, b^{\prime} ; m^{\prime}\right)$. Since $(X, \pi)=\left(X_{0}, \pi\right) \#(Y, \pi)$, it remains to see that the connecting points $\pi / \pi^{\prime} \times\{x\}$ in $X_{0}$ may be $\pi$-equivariantly isotoped into one of the tree factors. But this is clear, since $\operatorname{Fix}\left(X_{0}, \pi^{\prime}\right)$ is a disjoint union of isolated point and $2-$ spheres. If $\pi^{\prime}=1$ there is nothing to prove since we can move the orbit of $x$ around in the free part of the action. If $\pi^{\prime} \neq 1$, then the orbit of $x$ either lies in disjoint branches of the tree, or may be isotoped into a $\pi^{\prime}$-fixed 2 -sphere in one of the vertices. This shows that $(X, \pi)$ is a tree manifold.

\section{(1.E) Isotropy and fixed 2-spheres}

We will now discuss the isotropy subgroups and the existence of invariant 2spheres for the tree manifolds. A $\pi^{\prime}$-isotropy 2-sphere is a two-dimensional component of $X_{\left(\pi^{\prime}\right)}$. Suppose first that $\mathbb{T}_{0}$ is a homologically trivial tree with root $\mathbb{C} P^{2}(a, b)$, and let $X=X\left(\mathbb{T}_{0}\right)$. We may describe the rotation numbers at the other vertices algebraically by considering the three possible choices $p_{1}, p_{2}$, $p_{3}$ for attaching edges. With the conventions above, the possible new weights are $w_{1}=(a,-b), w_{2}=(-a, a-b)$ and $w_{3}=(-b, b-a)$. We can define the matrices

$$
K=\left(\begin{array}{rr}
1 & 0 \\
0 & -1
\end{array}\right), \quad L=\left(\begin{array}{rr}
-1 & 0 \\
1 & -1
\end{array}\right), \quad R=\left(\begin{array}{rr}
0 & -1 \\
-1 & 1
\end{array}\right)
$$

with the property that $w_{1}=K w, w_{2}=L w$ and $w_{3}=R w$, where $w=(a, b)$ and we perform the matrix multiplication with the weights as column vectors. The following result is clear. 
Lemma 1.8 Let $\Gamma \subset G L_{2}(\mathbf{Z})$ be the subgroup generated by the matrices $K$, $L$ and $R$. If $\mathbb{T}_{0}$ is a homologically trivial tree with root weights $w=(a, b)$, then the weights at the other vertices are of the form $\gamma \cdot w \bmod m$ for some $\gamma \in \Gamma$.

In principle, we could now determine all the possible isotropy subgroups in $X\left(\mathbb{T}_{0}\right)$ from this recipe. Notice the relations

$$
L^{k}=(-1)^{k}\left(\begin{array}{rr}
1 & 0 \\
-k & 1
\end{array}\right), \quad R^{k}=\left(\begin{array}{cc}
f_{k-1} & -f_{k} \\
-f_{k} & f_{k+1}
\end{array}\right)
$$

where $f_{k}$ denotes the $k^{t h}$ Fibonacci number starting with $f_{0}=0$ and $f_{1}=1$. Here is one simple observation.

Lemma 1.9 There is a homologically trivial tree $\mathbb{T}_{0}$ such that $X\left(\mathbb{T}_{0}\right)$ contains a $\pi^{\prime}$-isotropy 2-sphere for every subgroup $\pi^{\prime}$ of $\pi$.

Proof Starting with the weights $(1,0)$ and applying $L^{k}$ for $1 \leq k \leq m$ we will obtain the weights $w_{i}=\left(1,-m_{i}\right)$ for each divisor $m_{i}$ of $m$. Since the linear action $\mathbb{C} P^{2}\left(1,-m_{i} ; m\right)$ has a $\pi_{i}$-isotropy 2 -sphere (where $\left|\pi_{i}\right|=m_{i}$ ) we are done.

Remark 1.10 This result is of course very inefficient. We would like to prescribe a collection of subgroups $\left\{\pi_{1}, \ldots, \pi_{r}\right\}$ and find the minimal integer $n \geq 0$ such that there exists a homologically trivial action on $\#_{1}^{n} \mathbb{C} P^{2}$ with a $\pi^{\prime}$ isotropy 2-sphere exactly when $\pi^{\prime}=\pi_{i}$ for some $i, 1 \leq i \leq r$.

For the general case, we can analyse the isotropy groups and invariant 2 -spheres by considering the complement $\mathbb{T} \backslash \mathbb{T}_{0}$ as a disjoint union of weighted trees. The same process applies to each branch with respect to the stabilizer subgroup $\pi^{\prime}$ of the branch. To the operation of the matrices $K, L$, and $R$ above we add the operation of reduction mod $m^{\prime}$ as we enter the branch.

Lemma 1.11 Let $X(\mathbb{T})$ be a tree manifold. Then the weights at the root vertex determine the weights at all the vertices.

\section{(1.F) Permutation modules}

Let $X=X(\mathbb{T})$ be a tree manifold and consider the action of $\pi$ by permutations on the homology group

$$
H_{2}(X ; \mathbf{Z})=\bigoplus\left\{\mathbf{Z}\left[\pi / \pi_{\alpha}\right]^{k_{\alpha}}: \pi_{\alpha} \in \mathcal{S}\right\}
$$


We will assume that $X$ is an action on $\#_{1}^{n} \mathbb{C} P^{2}$ for some $n>0$ (thus eliminating a type II tree with only the root vertex). The set $\mathcal{S}$ is the set of stabilizer subgroups for elements in the standard basis $\left\{e_{1}, \ldots, e_{n}\right\}$ of $H_{2}(X ; \mathbf{Z})$. We observe that if $\{1\} \in \mathcal{S}$, then $\mathbf{Z}[\pi]$ is obtained by equivariant connected sum along a free orbit of points. At the other extreme, $\pi \in \mathcal{S}$ if and only if the homologically trivial part $\mathbb{T}_{0} \subset \mathbb{T}$ is non-empty (i.e. $\mathbf{T}$ has type I). The subgroup $\pi_{\alpha}=\pi$ contributes a summand $\mathbf{Z}^{k}$ to $H_{2}(X ; \mathbf{Z})$, where the Euler characteristic $\chi(\operatorname{Fix}(X, \pi))=k+2$. This means that in the equivariant connected sum $X\left(\mathbb{T}_{0}\right)$ there are exactly $k$ vertices.

Lemma 1.12 The proper, non-trivial, subgroups in the set $\mathcal{S}$ are exactly the set of subgroups $\left\{\pi^{\prime} \subset \pi\right\}$ for which (i) $\operatorname{Fix}\left(X, \pi^{\prime}\right)$ contains a 2-sphere at a vertex $v_{j}$, and (ii) there is an edge $e \in \mathbf{T}$ with $\partial_{0} e=v_{i}$ and $\partial_{1} e=v_{j}$ such that $m_{i}>m_{j}=\left|\pi^{\prime}\right|$.

Proof Suppose that $1 \neq \pi^{\prime} \in \mathcal{S}$. Then there exists a vertex $v_{j}$ with stabilizer $\pi^{\prime}=\pi_{j}$ of order $m_{j}$. The conditions follow easily.

We will be interested in the maximal elements of the set $\mathcal{S}$ under the partial ordering by inclusion on subgroups. Recall that disjoint subgroups are those which intersect only at the identity element.

Lemma 1.13 Let $X=X(\mathbb{T})$ be a tree manifold diffeomorphic to $\#_{1}^{n} \mathbb{C} P^{2}$ for some $n>0$. Then the set $\mathcal{S}$ of stabilizer subgroups for the permutation modules in $H_{2}(X ; \mathbf{Z})$ has the unique maximal element $\{\pi\}$ if $\mathbf{T}$ has type $I$, and otherwise $\mathcal{S}$ has at most two maximal elements, and these are disjoint.

Proof Of course if $\mathbf{T}$ has type I, then the root vertex is $\pi$-fixed and the homologically trivial part $\mathbb{T}_{0}$ is non-empty, so we get copies of $\mathbf{Z}$ in the permutation module. If $\mathbf{T}$ has type II, then there are no $\pi$-fixed vertices except the root vertex, but this vertex is of the form $S^{4}(a, b ; m)$ so its invariant 2 -spheres are homologically zero. However, the equivariant connected sum must be performed at a $\pi$-orbit of connecting points, either in the free part of the action or in one of the (at most) two singular 2-spheres. The isotropy groups of these singular 2 -spheres in $S^{4}$ are the maximal elements in $\mathcal{S}$, which are necessarily disjoint subgroups of $\pi$.

Remark 1.14 Since the linear actions $\mathbb{C} P^{2}(a, b)$ have at most three non-trivial proper isotropy groups, the realizable sets $\mathcal{S}$ of stabilizer subgroups have an 
additional property for type I tree manifolds with the root vertex as the unique $\pi$-fixed vertex: $\mathcal{S}$ contains at most three maximal proper subgroups, $\pi_{1}, \pi_{2}$ and $\pi_{3}$, and $\pi_{i} \cap \pi_{j}=\{1\}$ for $i \neq j$.

We now have two stability results for realizing permutation modules by tree manifolds.

Theorem 1.15 Let $\mathcal{S}$ denote a set of subgroups with $\pi \in \mathcal{S}$. There exists an integer $N=N(\mathcal{S}) \leq m$, such that any permutation module

$$
\bigoplus\left\{\mathbf{Z}\left[\pi / \pi_{\alpha}\right]^{k_{\alpha}}: \pi_{\alpha} \in \mathcal{S}\right\}
$$

with multiplicity $k_{0}>N$ for the trivial representation $\mathbf{Z}=\mathbf{Z}[\pi / \pi]$ is realizable by a tree manifold $X(\mathbb{T})$.

Proof We start with any homologically trivial action that contains an isotropy 2 -sphere for each $\pi_{\alpha} \in \mathcal{S}$. This can be achieved by taking enough vertices in $\mathbb{T}_{0}$, as shown above. We then use these $\pi_{\alpha}$-isotropy two spheres as the attaching spheres for $k_{\alpha}$ branches of the form $\pi \times_{\pi_{\alpha}} \mathbb{C} P^{2}$.

For type II trees we assume stability at both maximal elements of the set of stabilizer subgroups.

Theorem 1.16 Let $\mathcal{S}$ denote a set of subgroups with at most two maximal elements $\left\{\pi_{1}, \pi_{2}\right\} \in \mathcal{S}$. If $\mathcal{S}$ has two maximal elements, suppose that they are disjoint. Then there exists an integer $N=N(\mathcal{S}) \leq m$ such that any permutation module

$$
\bigoplus\left\{\mathbf{Z}\left[\pi / \pi_{\alpha}\right]^{k_{\alpha}}: \pi_{\alpha} \in \mathcal{S}\right\}
$$

with multiplicities $k_{i}>N$, for $i=1,2$, is realizable by a type II tree manifold $X(\mathbb{T})$.

Proof In the type II case we must attach a $\pi_{i}$-homologically trivial branch to each of the $\pi_{i}$-isotropy 2 -spheres in the root $S^{4}$. If we take sufficiently many vertices in these branches we can obtain isotropy 2 -spheres for all the remaining subgroups of $\mathcal{S}$. This is because each $\pi_{\alpha} \in \mathcal{S}$ is contained in one of the maximal elements $\pi_{1}$ or $\pi_{2}$. Then we attach orbits of the form $\pi_{i} \times_{\pi_{\alpha}} \mathbb{C} P^{2}$ in the $\pi_{i}$-branch. This realizes the module $\mathbf{Z}\left[\pi_{i} / \pi_{\alpha}\right]$ in the homology of the branch, but the $\pi$-equivariance gives us the module

$$
\operatorname{Ind}_{\pi_{i}}^{\pi}\left(\mathbf{Z}\left[\pi_{i} / \pi_{\alpha}\right]\right)=\operatorname{Ind}_{\pi_{i}}^{\pi}\left(\operatorname{Ind}_{\pi_{\alpha}}^{\pi_{i}}(\mathbf{Z})\right)=\operatorname{Ind}_{\pi_{\alpha}}^{\pi}(\mathbf{Z})=\mathbf{Z}\left[\pi / \pi_{\alpha}\right]
$$

in the homology of $X$. 
Remark 1.17 The method of proof shows that any $N \geq m$ works. More precisely, we could take $N=\max \left\{\left|\pi_{\alpha}\right|: \pi_{\alpha} \in \mathcal{S}\right\}$. These estimates are probably far from "best possible".

Example 1.18 The permutation module $\mathbf{Z}\left[C_{15}\right] \oplus \mathbf{Z}\left[C_{21}\right] \oplus \mathbf{Z}\left[C_{35}\right]$, with $\pi=$ $C_{105}$, is not realizable as $H_{2}(X ; \mathbf{Z})$ for any equivariant connected sum of linear actions. In this case $\mathcal{S}$ contains the three maximal elements $\left\{C_{3}, C_{5}, C_{7}\right\}$. However, the module $\mathbf{Z} \oplus \mathbf{Z}\left[C_{15}\right] \oplus \mathbf{Z}\left[C_{21}\right] \oplus \mathbf{Z}\left[C_{35}\right]$ is realizable, starting from the root $\mathbb{C} P^{2}(10,3 ; 105)$.

\section{Equivariant moduli spaces}

In [1] the theory of Yang-Mills moduli spaces (3], 5]) was adapted to the equivariant setting. We give an informal sketch of the main features and refer to these sources for details. Let $P \rightarrow X$ be a principal $S U(2)$-bundle over a smooth, closed, oriented, simply-connected 4-manifold $X$. Let $\mathcal{A}(P)$ denote the space of $S U(2)$ connections on $P$, and $\mathcal{B}(P)=\mathcal{A}(P) / \mathcal{G}(P)$ the quotient space of connections by the action of the gauge group $\mathcal{G}(P)$. If we fix a Riemannian metric on $X$, we can decompose the space of Lie algebra valued forms

$$
\Omega^{2}(X ; \operatorname{ad} P)=\Omega_{+}(X ; \operatorname{ad} P) \oplus \Omega_{-}(X ; \operatorname{ad} P)
$$

into eigenspaces of the $*$-operator. The curvature operator

$$
F: \mathcal{A}(P) \rightarrow \Omega^{2}(X ; \text { ad } P)
$$

is gauge invariant and decomposes as $F=F^{+} \oplus F^{-}$. We can define the self-dual Yang-Mills moduli space

$$
\mathcal{M}(P)=\left\{[A] \in \mathcal{B}(P) \mid F_{A}^{-}=0\right\}
$$

Now suppose that we have a smooth, orientation-preserving action $(X, \pi)$. We fix a real analytic structure on $X$ compatible with its smooth structure and the given $\pi$-action, and a real analytic $\pi$-invariant metric on $X$ (see [13]). Since action of $\pi$ on $X$ preserves the orientation, and the bundle $P$ is classified by $c_{2}(P) \in H^{4}(X ; \mathbf{Z})$, for each element $g \in \pi$, we can find a (generalized) bundle map $\varphi_{g}: P \rightarrow P$ such that the diagram

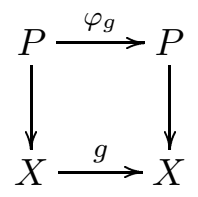


commutes. Let $\mathcal{G}(\pi):=\left\{\varphi_{g} \mid g \in \pi\right\}$ denote the group of generalized gauge transformations. Since the indeterminacy in the choice of $\varphi_{g}$ is an element of $\mathcal{G}$, we have a short exact sequence

$$
1 \rightarrow \mathcal{G} \rightarrow \mathcal{G}(\pi) \rightarrow \pi \rightarrow 1
$$

of groups, and a well-defined $\pi$-action on $\mathcal{B}$. The gauge-invariance of the curvature operator implies that the map $F$ is also $\pi$-equivariant, and hence we obtain a natural $\pi$-action $(\mathcal{M}, \pi)$ on the Yang-Mills moduli space.

Without further modifications, the moduli space could be a very singular object. The index $\delta_{A}$ of its fundamental elliptic complex

$$
\Omega^{0}(X ; \operatorname{ad} P) \stackrel{d_{A}}{\longrightarrow} \Omega^{1}(X ; \operatorname{ad} P) \stackrel{d_{A}^{-}}{\longrightarrow} \Omega_{-}(X ; \operatorname{ad} P)
$$

is called the formal dimension of the moduli space at $[A] \in \mathcal{M}$, but it need not be its geometric dimension. The operator $d_{A}$ is the covariant derivative associated to the connection $A$, and the operator $d_{A}^{-}$is the linearization of the curvature.

The homology groups $H_{A}^{i}, 0 \leq i \leq 2$, of the fundamental elliptic complex are finite dimensional real vector spaces, whose dimensions $h^{i}(A)=\operatorname{dim} H_{A}^{i}$ appear in the formula

$$
\delta_{A}=h^{1}(A)-h^{0}(A)-h^{2}(A) .
$$

By the Atiyah-Singer Index Theorem, the formal dimension has a purely topological expression $\delta_{A}=-8 c_{2}(P)-3\left(1-b_{2}^{-}\right)$, where $b_{2}^{-}=\operatorname{dim} H_{-}^{2}(X ; \mathbb{R})$. In particular, it is independent of the choice of base point $[A]$. A necessary condition for $P$ to admit any self-dual connections is $c_{2}(P) \leq 0$, and we will only use the case $c_{2}=-1$ and $b_{2}^{-}=0$ in this paper.

In the equivariant setting, the elliptic complex inherits an action of the stabilizer $\mathcal{G}_{A}(\pi) \leq \mathcal{G}$ of the connection under the generalized gauge group action. The stabilizer sits in an exact sequence

$$
1 \rightarrow \Gamma_{A} \rightarrow \mathcal{G}_{A}(\pi) \rightarrow \pi_{A} \rightarrow 1
$$

where $\pi_{A}$, by definition, is the image of $\mathcal{G}_{A}(\pi)$ in $\pi$. Here $\Gamma_{A}$ is the stabilizer of $A$ in $\mathcal{G}$, and there are just two possibilities: $\Gamma_{A}=\{ \pm 1\}$ if $A$ is irreducible, or $\Gamma_{A}=S^{1}$ if $A$ is reducible. The latter holds when the structural group $S U(2)$ of $P$ reduces to $S^{1}$, or equivalently, when the associated complex vector bundle $E \rightarrow X$ splits as $E=L \oplus L^{-1}$ for some complex line bundle over $X$.

The Kuranishi method gives finite dimensional "local charts"

$$
\phi_{A}: H_{A}^{1} \rightarrow H_{A}^{2}
$$


at each connection $[A] \in \mathcal{M}$, where $\phi_{A}$ is a $\mathcal{G}_{A}(\pi)$-equivariant smooth map with $\left(d \phi_{A}\right)_{0}=0$. Then in a neighbourhood of $[A]$, the moduli space $\mathcal{M}$ is locally isomorphic to $\phi_{A}^{-1}(0) / \Gamma_{A}$.

\section{(2.A) Equivariant general position}

In [11, an equivariant perturbation of the Yang-Mills equations was constructed (based on the method of Bierstone [1]) to obtain an equivariant general position moduli space of self-dual connections $(\mathcal{M}, \pi)$ ). After perturbation, the equivariant moduli space is locally described by finite dimensional charts $\phi_{A}: H_{A}^{1} \rightarrow H_{A}^{2}$ as above, which are in Bierstone general position with respect to the $\pi_{A}$ action (if $A$ is irreducible) or the $S^{1} \times \pi_{A}$ action (if $A$ is reducible). If $H_{A}^{2}=0$ after the perturbation, then the moduli space is equivariantly transverse at $[A]$. We obtain the following good properties:

(i) $(\mathcal{M}, \pi)$ is an equivariantly Whitney stratified space [14 with an effective $\pi$-action and open smooth manifold strata

$$
\mathcal{M}_{\left(\pi^{\prime}\right)}^{*}=\left\{x \in \mathcal{M}^{*} \mid \pi_{x}=\pi^{\prime} \subseteq \pi\right\},
$$

where $\mathcal{M}^{*} \subseteq \mathcal{M}$ is the subset of irreducible connections (up to gauge equivalence).

(ii) For $\pi^{\prime} \subseteq \pi$ each component of the fixed point set $\operatorname{Fix}\left(\mathcal{M}^{*}, \pi^{\prime}\right)$ is the moduli space of $\pi^{\prime}$-invariant connections on $P$ with respect to a $\pi^{\prime}-S U(2)$ bundle structure on $P$ (compare [9], 8]).

(iii) The strata have topologically locally trivial equivariant cone bundle neighbourhoods in $(\mathcal{M}, \pi)$.

(iv) $(\mathcal{M}, \pi)$ has an equivariant compactification $(\overline{\mathcal{M}}, \pi)$. When $c_{2}(P)=-1$, and $X$ has positive definite intersection form,

$$
(\overline{\mathcal{M}}, \pi)=\left(\mathcal{M} \cup \tau\left(X \times\left[0, \lambda_{0}\right)\right), \pi\right)
$$

where the ideal boundary $\partial \overline{\mathcal{M}}=\tau(X \times 0)$ is the Taubes embedding of $X$ as the set of ideal "highly-concentrated" connections. Under this embedding, the boundary of $\overline{\mathcal{M}}$ has a smooth equivariant collar neighbourhood, which is $\pi$-equivariantly diffeomorphic to $X \times\left[0, \lambda_{0}\right)$ with the product action $\left(0<\lambda_{0} \ll 1\right)$.

(v) The dimensions of the strata $\mathcal{M}_{\left(\pi^{\prime}\right)}$ can be computed as the index of the $\pi^{\prime}$-fixed set of the fundamental elliptic complex. A stratum $\mathcal{M}_{\left(\pi^{\prime}\right)}$ is non-empty whenver its formal dimension is positive. In particular, when $c_{2}(P)=-1$, the free stratum $\mathcal{M}_{(e)}^{*}$ is a smooth, non-compact 5 dimensional manifold. We may assume that the free stratum is connected. 
Remark 2.2 For the case $c_{2}=-1$ and $X$ has positive definite intersection form, the moduli space is equivariantly transverse on the subspace $\mathcal{M}_{\lambda_{0}}:=$ $\mathcal{M}^{*} \cap \tau\left(X \times\left[0, \lambda_{0}\right)\right)$. of highly concentrated connections [10, Section 9].

From now on we assume that $X$ is a closed, smooth, simply-connected $4-$ manifold with positive definite intersection form. In other words, we assume that $X \simeq \#_{1}^{n} \mathbb{C} P^{2}$ for some $n>0$. We also restrict attention to the equivariant moduli space $(\mathcal{M}, \pi)$ for $c_{2}=-1$, and let $\pi=C_{m}$ for $m$ odd. Under these assumptions, the moduli space has a number of additional properties. Most of these properties were established in [12] for the special case when the induced $\pi$-action on homology is trivial. We will adapt the statements to remove this assumption, and indicate where the proofs need to be generalized.

\section{(2.B) Reducible connections}

For $X \simeq \#_{1}^{n} \mathbb{C} P^{2}$ we fix a standard basis $\left\{e_{1}, \ldots, e_{n}\right\}$ for $H_{2}(X ; \mathbf{Z})$ so that $Q_{X}\left(e_{i}, e_{j}\right)=\delta_{i j}$.

Lemma 2.3 [5, 2.2.6] Let $P \rightarrow X$ be a principal $S U(2)$-bundle with $c_{2}(P)=$ -1 , where $X \simeq \#_{1}^{n} \mathbb{C} P^{2}$. There is a $\pi$-equivariant bijection $e_{i} \leftrightarrow\left[D_{i}\right]$ between the set $\left\{e_{1}, e_{2}, \ldots, e_{n}\right\}$ of standard basis elements for $H_{2}(X)$ and the set $\mathcal{R}:=\left\{\left[D_{1}\right],\left[D_{2}\right], \ldots,\left[D_{n}\right]\right\}$ of gauge equivalence classes of reducible $S U(2)-$ connections on $P$.

Proof The reducible connections correspond to splittings $E=L \oplus L^{-1}$, for some complex line bundle $L \rightarrow X$. Such splitting exist if and only if $c_{2}(E)=$ $-x^{2}$ for some $x \in H^{2}(X ; \mathbf{Z})$, since any such class determines a line bundle $L$ with $c_{1}(L)=x$. Since the only solutions for the equation $x^{2}=1$ (up to \pm 1 ) come from the Poincaré duals $\hat{e}_{i}$ of the basis elements $e_{i}$, we have a bijection between the two sets. However, we have a $\pi$-action on the set of reducibles and on the homology classes, and we must check that the actions correspond. Let $\pi_{i}$ denote the stabilizer of $e_{i}$ under the $\pi$-action on homology. Then, by the same proof as [12, Corollary 1], there exists a $\pi_{i}$-equivariant $S^{1}$ bundle $L_{i} \rightarrow X$ such that $c_{1}\left(L_{i}\right)=\hat{e}_{i}$. Since an $S^{1}$-bundle has a unique self-dual connection $\left[D_{i}\right]$, up to gauge equivalence, we see that $\left[D_{i}\right]$ is $\pi_{i}$-invariant. This shows that $\left[g^{*} D_{i}\right]=\left[D_{j}\right]$ if and only if $c_{1}\left(g^{*} L_{i}\right)=L_{j}$, which holds if and only if $g^{*}\left(e_{i}\right)=e_{j}$. Therefore the actions agree.

The most striking feature of this $c_{2}=-1$ equivariant moduli space is the existence of equivariantly transversal local charts at each of the reducible connections. Here we generalize [12, Theorem 15]. 
Theorem 2.4 Let $P \rightarrow X$ be a principal $S U(2)$-bundle with $c_{2}(P)=-1$, where $X \simeq \#_{1}^{n} \mathbb{C} P^{2}$. Then for each reducible connection $[D]$, there is a $\pi_{D^{-}}$ invariant neighbourhood $\mathcal{N}_{D}$ of $[D]$ in $(\mathcal{M}, \pi)$ on which the moduli space is equivariantly transverse. The stratified space $\left(\mathcal{N}_{D},[D]\right)$ is $\pi_{D}$-equivariantly homeomorphic to the cone over some linear action of $\pi_{D}$ on $\mathbb{C} P^{2}$. Away from the cone point, these two stratified spaces are equivariantly diffeomorphic.

Proof The proof of this result in the homologically trivial case in 12, Section 4] is fairly complicated, but the changes needed to allow a non-trivial action on homology are minimal. The point is that the set of reducibles is finite, so that a $\pi$-invariant neighbourhood of a reducible $[D]$ has the form $\mathcal{U}=\pi \times_{\pi_{D}} \mathcal{N}$ for some $\pi_{D}$-invariant neighbourhood $\mathcal{N}$ of $[D]$. We may assume that $\mathcal{N} \cap g(\mathcal{N})=$ $\emptyset$ if $g \notin \pi_{D}$, so the quotient group $\pi / \pi_{D}$ permutes a set of disjoint copies of $\mathcal{N}$ freely and transitively. The perturbations needed to achieve $\pi_{D^{-}}$equivariant transversality in a neighbourhood of $[D]$ (exactly as carried out in [12, Section 4]) can be extended $\pi$-equivariantly to achieve transversality at each reducible in the $\pi$-orbit of $[D]$. Since the set of reducibles is a disjoint union of $\pi$-orbits, and the perturbations are local, we can achieve transversality at each orbit separately.

Remark 2.5 We have shown that $H_{D}^{2}=0$ for each reducible connection $[D]$. The local cone $\mathcal{N}_{D}$ is just the quotient of $H_{D}^{1}=\mathbb{C}\left(\chi_{1}\right) \oplus \mathbb{C}\left(\chi_{2}\right) \oplus \mathbb{C}\left(\chi_{3}\right)$ by the stabilizer $\Gamma_{D}=S^{1}$ acting diagonally as complex multiplication. The weights $\chi_{i}, 1 \leq i \leq 3$ are linear characters of $\pi_{D}$, and the subspaces $\mathbb{C}\left(\chi_{i}\right)$ are just the fixed sets $\operatorname{Fix}\left(H_{D}^{1}, \pi_{D}\left(\chi_{i}\right)\right)$, where $\pi_{D}\left(\chi_{i}\right):=\left\{\left(g, \chi_{i}\left(g^{-1}\right) \mid g \in \pi_{D}\right\} \subset \pi_{D} \times S^{1}\right.$. We call the boundary $\ell(D):=\partial \mathcal{N}_{D}$ the link of the reducible connection.

\section{(2.C) Orientation}

The Yang-Mills moduli space inherits an orientation from that of $X$ (see [4], [5. Section 5.4]). Donaldson shows that the real determinant line bundle $\Lambda(P)$ associated to the elliptic complex

$$
\Omega_{-}^{*}(\operatorname{ad} P): \quad 0 \rightarrow \Omega^{0}(\operatorname{ad} P) \rightarrow \Omega^{1}(\operatorname{ad} P) \rightarrow \Omega_{-}(\operatorname{ad} P) \rightarrow 0
$$

has a canonical trivialization over $\mathcal{B}$ which induces the given orientation on $X$ times the inward pointing normal, where $X$ is embedded as the Taubes boundary in $\mathcal{M}$. We call this the canonical orientation of $\mathcal{M}$.

Lemma 2.6 (see [12, Lemma 8]) Let $\pi=C_{m}$ for $m$ odd, and for any subgroup $\pi^{\prime}$ of $\pi$ let $\mathcal{C} \subset \mathcal{M}_{\left(\pi^{\prime}\right)}^{*}$ be a connected component. Then the canonical orientation on $\mathcal{M}$ induces a preferred orientation on the smooth manifold $\mathcal{C}$. 
Proof If $[A] \in \mathcal{C}$, we can split the elliptic complex

$$
\Omega_{-}^{*}(\operatorname{ad} P)=\Omega_{-}^{*}(\operatorname{ad} P)^{\pi^{\prime}} \oplus\left[\Omega_{-}^{*}(\operatorname{ad} P)^{\pi^{\prime}}\right]^{\perp}
$$

into a fixed subcomplex and a perpendicular complex. It follows that the line bundle $\Lambda(P)=\Lambda_{t(\mathcal{C})} \otimes \Lambda_{n(\mathcal{C})}$, where $\Lambda_{t(\mathcal{C})}$ is the determinant line bundle of $\Omega_{-}^{*}(\operatorname{ad} P)^{\pi^{\prime}}$ and $\Lambda_{n(\mathcal{C})}$ is for the complementary part of $\Omega_{-}^{*}(\operatorname{ad} P)$. Since $\pi$ is odd order cyclic, the action of $\pi^{\prime}$ on $\left[\Omega_{-}^{*}(\operatorname{ad} P)^{\pi^{\prime}}\right]^{\perp}$ induces a complex structure, and hence a preferred orientation on $\Lambda_{n(\mathcal{C})}$. Then the canonical orientation on $\mathcal{M}$ induces an orientation on $\Lambda_{t(\mathcal{C})}$ as well. However, the moduli space is locally modelled on a the zero set of a smooth map $f: H_{A}^{1} \rightarrow H_{A}^{2}$ in Bierstone general position. Furthermore, the fixed set $\left(\text { Coker } d f_{0}\right)^{\pi^{\prime}}=0$ and Ker $d f_{0}$ (which is fixed under $\pi^{\prime}$ ) is the tangent space to the manifold stratum $\mathcal{C}$ at $[A]$. Therefore $C$ has a preferred orientation.

We may apply this to the free stratum, and consider the copies of $\mathbb{C} P^{2}$ which bound $\pi_{D}$-invariant neighbourhoods $\mathcal{N}_{D}$ of each of the reducible connections $[D] \in \mathcal{M}$. Note that we have an $S^{1}$ determinant line bundle $\mathcal{L}_{\pi^{\prime}}$ over $\operatorname{Fix}\left(\mathcal{M}, \pi^{\prime}\right)$ equal to the top exterior power with respect to the complex structure on $\left[\Omega_{-}^{*}(\operatorname{ad} P)^{\pi^{\prime}}\right]^{\perp}$.

Lemma 2.7 4, Example 4.3] The induced orientation on each copy of $\mathbb{C} P^{2}=$ $\partial \mathcal{N}_{D}$ linking a reducible connection $[D]$ is the standard complex orientation.

Proof This amounts to the statement that the preferred complex line bundle $\mathcal{L}_{\pi_{D}}$, restricted to the link of each reducible fixed by $\pi_{D}$, is just the Hopf bundle over $\mathbb{C} P^{2}$.

Corollary 2.8 Let $\pi_{D}$ be the isotropy group of a reducible connection $[D]$, and let $\pi^{\prime} \subseteq \pi_{D}$.

(i) Then there is no continuous path $\gamma:[0,1] \rightarrow \overline{\mathcal{M}_{\left(\pi^{\prime}\right)}}$ such that $\gamma(0)=$ $\gamma(1)=[D]$, with $\gamma(0,1) \cap \mathcal{N}_{D}$ a disjoint union of one-dimensional strata.

(ii) Suppose that $\left[D^{\prime}\right]=g^{*}[D] \neq[D]$ for some $g \in \pi$. Then there is no continuous path $\gamma:[0,1] \rightarrow \overline{\mathcal{M}_{\left(\pi^{\prime}\right)}}$ such that $\gamma(0)=[D], \gamma(1)=\left[D^{\prime}\right]$, with $\gamma(0,1) \cap \mathcal{N}_{D}$ and $\gamma(0,1) \cap \mathcal{N}_{D^{\prime}}$ a disjoint union of one-dimensional strata.

Proof The argument is given in the proof of [12. Theorem C, page 729], based on the orientability of the $\pi_{D}$-fixed strata in the moduli space (see Lemma 2.6). 
For part (i), suppose that there exists a such a closed path $\gamma$ in $\operatorname{Fix}\left(\mathcal{M}, \pi_{D}\right)$, intersecting the cone $\mathcal{N}_{D}$ in two distinct one-dimensional strata with isotropy groups $\pi_{D}\left(\chi_{i}\right), \pi_{D}\left(\chi_{j}\right)$ respectively, in the $\pi_{D} \times S^{1}$ action of the local model. But the $S^{1}$ determinant line bundle $\mathcal{L}_{\pi_{D}}$ restricted to these two strata is positively oriented on each stratum by the complex structure. However the $S^{1}$ determinant line bundle extends over $\operatorname{Fix}\left(\mathcal{M}, \pi_{D}\right)$, and so over $\gamma$ giving a contradiction. This shows that such a loop $\gamma$ can't exist.

For part (ii) we use the orientation-preserving action of $g \in \pi$ to identify the $S^{1}$ determinant line bundles $\mathcal{L}_{\pi_{D}}$ and $\mathcal{L}_{\pi_{D^{\prime}}}$ at the distinct reducibles $[D]$ and $\left[D^{\prime}\right]$. Now the assumption that the $S^{1}$ determinant line bundle extends over the path $\gamma$ again gives a contradiction.

The existence of topologically locally trivial cone bundle neighbourhoods for the strata in $\mathcal{M}$ allows the possibility of transporting rotation number information from $X$ to the links of the reducible connections. If $x \in X$ lies in $\operatorname{Fix}\left(X, \pi^{\prime}\right)$ then $\left(T_{x} X, \pi^{\prime}\right)$ splits into a trivial $\pi^{\prime}$-representation tangent to the fixed set, and a complementary normal isotropy representation $V_{x}$. If $x \in \operatorname{Fix}\left(X, \pi^{\prime}\right)$ is an isolated fixed point, then $V_{x}$ is just the isotropy representation $\left(T_{x} X, \pi^{\prime}\right)$ whose eigenvalues are the rotation numbers $\left(\bmod \left|\pi^{\prime}\right|\right)$ at $x$. Let $\mathcal{M}_{\lambda_{0}}=\mathcal{M}^{*} \cap$ $\tau\left(X \times\left[0, \lambda_{0}\right)\right)$ denote the intersection of the Taubes collar with the subspace of irreducible connections. We will study the strata containing a singular set of the form $F \times\left(0, \lambda_{0}\right) \subset \mathcal{M}_{\lambda_{0}}$, where $F \subset \operatorname{Sing}(X, \pi)$.

Lemma 2.9 11, Corollary 3.2] Let $F \subset X_{\left(\pi^{\prime}\right)}$ be a connected component with normal isotropy representation $V_{x} \subset T_{x} X$ at $x \in F$. Then there exists a smooth manifold stratum $\mathcal{C} \subset \mathcal{M}_{\left(\pi^{\prime}\right)}^{*}$, such that $\mathcal{C} \cap \mathcal{M}_{\lambda_{0}}=F \times\left(0, \lambda_{0}\right)$. In addition, the cone bundle neighbourhood for $\mathcal{C}$ is a $\pi^{\prime}$-equivariant disk bundle with normal isotropy representation $V_{x}$.

It follows that such a stratum carries a preferred orientation for its normal disk bundle in $\mathcal{M}^{*}$. This can sometimes be used to determine its closure.

Lemma 2.10 Suppose that $\pi^{\prime} \neq \pi$ and that $x$ is an isolated fixed point in $\operatorname{Fix}\left(X, \pi^{\prime}\right)$. Then the closure of the connected 1 -dimensional stratum $\mathcal{C}$ in $\mathcal{M}_{\left(\pi^{\prime}\right)}^{*}$ containing $\{x\} \times\left(0, \lambda_{0}\right)$ either ends at another isolated fixed point $y \neq x$ in $\operatorname{Fix}\left(X, \pi^{\prime}\right)$, or ends at a reducible connection.

Proof Under the given assumptions, $\mathcal{C}$ is a smooth non-compact 1 -dimensional manifold with one limiting endpoint at $x$. If its other limiting endpoint 
$y \in X$ lies in the Taubes boundary, then $y$ must clearly be isolated and distinct from $x$. Suppose that $[A] \in \mathcal{M}^{*}$ is the other limiting endpoint in $\overline{\mathcal{C}}$. Then, by general position, $[A]$ must have a larger isotropy subgroup $\pi_{A} \neq \pi^{\prime}$. Therefore the full orbit of $\mathcal{C}$ under the $\pi_{A}$ action must also have $[A]$ as a limiting endpoint, and for any $g \in \pi_{A}, g \notin \pi^{\prime}$, the union $\overline{\mathcal{C}} \cup g \cdot \overline{\mathcal{C}}$ gives a continuous path from $x$ to $g x \neq x$. However, by transporting the preferred orientation for $T_{x} X$ along this path, we conclude that the rotation numbers at $g x \in X$ are $(a,-b)$, opposite to those at $x$. This is a contradiction, since the $\pi$-action on $X$ is orientationpreserving, so we have eliminated the possibility that $\mathcal{C}$ has a limiting endpoint in $\mathcal{M}^{*}$. But $\mathcal{C}$ is a smooth 1 -manifold, so it has two ends, and $\overline{\mathcal{C}}$ must have another limit point in $\overline{\mathcal{M}}$. It follows that $\overline{\mathcal{C}}$ contains a reducible connection

Corollary 2.11 11, Corollary 3.3] Suppose that $x$ is an isolated fixed point in $\operatorname{Fix}\left(X, \pi^{\prime}\right)$ with rotation numbers $(a, b)$. If the cancelling pair $(a,-b)$ of rotation numbers does not occur in $(X, \pi)$, then the closure of the connected 1 -dimensional stratum $\mathcal{C}$ in $\mathcal{M}_{\left(\pi^{\prime}\right)}^{*}$ containing $\{x\} \times\left(0, \lambda_{0}\right)$ ends at a reducible connection.

Proof If $\pi^{\prime}=\pi$ the smooth 1 -manifold $\mathcal{C}$ can't have a limiting endpoint in $\mathcal{M}^{*}$, by general position, so it must give a smooth path to a reducible connection. For the case $\pi^{\prime} \neq \pi$, if the closure of $\mathcal{C}$ doesn't contain a reducible, then Lemma 2.10, asserts that $\overline{\mathcal{C}}$ gives a smooth path from $x \in X$ to another isolated $\pi^{\prime}$-fixed point $y \in X$. But then Lemma 2.9 shows that the rotation numbers at $y$ are $(a,-b)$, which is a contradiction.

Remark 2.12 A smooth path $\gamma:[0,1] \rightarrow \overline{\mathcal{M}}$ emerging from an isolated $\pi^{\prime}$ fixed point on $X$, and ending at a reducible, is unique (up to re-parametrization) since for $0<t<1$, the path $\gamma(t)$ is contained in a 1 -dimensional smooth manifold component of the stratum $\mathcal{M}_{\left(\pi^{\prime}\right)}^{*}$. We point out another situation where such a path must exist. Recall that $\mathcal{R}$ denotes the set of reducible connections.

Corollary 2.13 [12, Lemma 17] If $\operatorname{Fix}\left(\mathcal{R}, \pi^{\prime}\right) \neq \emptyset$, for some $\pi^{\prime} \neq 1$ and $x \in X$ is an isolated $\pi^{\prime}$-fixed point, then the closure of the 1-dimensional stratum in $\mathcal{M}_{\left(\pi^{\prime}\right)}^{*}$ containing $\{x\} \times\left(0, \lambda_{0}\right)$ ends at a reducible connection.

Proof We remark that is possible for all the reducible connections to have have trivial stabilizer. For example, we may start with $S^{4}(a, b)$ and form the equivariant connected sum with copies of $\mathbb{C} P^{2}$ along a free orbit. Then there exists a fixed arc in $\overline{\mathcal{M}}^{*}$ whose endpoints are two isolated fixed points on $X$. 
Suppose, if possible, that there exists a $\pi^{\prime}$-fixed smooth path $\gamma$ joining two isolated $\pi^{\prime}$-fixed points $x_{1}, x_{2}$ in $X$. Then the rotation numbers at $x_{1}$ and $x_{2}$ form a cancelling pair $(a, b)$ and $(a,-b)$. By Lemma 2.9 there is a neighbourhood of $\gamma$ in $\overline{\mathcal{M}}$ which is cut out $\pi$-equivariantly transversely. We may now pick a non-trivial subgroup $C_{p} \subset \pi^{\prime}$ and perturb the equations defining the moduli space into $C_{p}$-general position. The perturbation can be chosen to be the identity on this neighbourhood, so the arc $\gamma$ does not change. Since $\operatorname{Fix}\left(\mathcal{R}, \pi^{\prime}\right) \neq \emptyset$, we have a non-zero trace for the $C_{p}$-action on $H_{2}(X ; \mathbf{Z})$ and therefore $\chi\left(\operatorname{Fix}\left(X, C_{p}\right)\right) \geq 3$. It follows that there exists $x_{3} \in \operatorname{Fix}\left(X, C_{p}\right)$ with $x_{3} \neq x_{1}, x_{2}$. Now the argument proceeds exactly as in [12, page 727].

\section{(2.D) Fixed sets in the closure of the free stratum}

For our applications to group actions on $\#_{1}^{n} \mathbb{C} P^{2}$ we only need to study the closure $\overline{\mathcal{M}_{(e)}^{*}}$ of the free stratum of the moduli space. By general position (see Section (2.A), and the good cone structure at the reducibles (Theorem 2.4) the closures of any singular strata of dimension $\geq 5$ are disjoint from the closure of the free stratum. This means that $\overline{\mathcal{M}_{(e)}^{*}}$ is the union of the free stratum with (i) the reducibles and (ii) some singular strata of dimension one or three. We are interested in knowing more about the closures of the singular strata inside $\overline{\mathcal{M}_{(e)}^{*}}$.

Lemma 2.14 [12, Lemma 12] Let $C \subseteq \mathcal{M}_{\left(\pi^{\prime}\right)}^{*}$ be a non-compact, connected component with $\pi^{\prime} \neq 1$. If $C$ is non-empty, then $\operatorname{dim} C<5$. If $\operatorname{dim} C=3$, then the closure $\bar{C} \subset \overline{\mathcal{M}}$ must intersect the Taubes boundary $\partial \overline{\mathcal{M}}=X \times\{0\} \subset \overline{\mathcal{M}}$.

Proof For $\operatorname{dim} C \geq 5$, the proof given in [12, Lemma 12] applies without change: assuming $C$ is non-empty leads to a contradiction. If $\operatorname{dim} C=3$ and $\pi^{\prime} \neq \pi$ we assume, if possible, that the closure $\bar{C}$ is formed by adjoining some one-dimensional strata in $\mathcal{M}^{*}$ (with larger isotropy), together with some $\pi^{\prime}$ fixed reducible connections. Now we remove the intersection of $\bar{C}$ with the interiors of the cones $\mathcal{N}_{D}$ around each of these reducibles. We then obtain a compact $\pi^{\prime}$-fixed set $(\mathcal{C}, \partial \mathcal{C})$ in $\mathcal{M}^{*}$, bounding a collection $F$ of 2 -dimensional $\pi^{\prime}$-fixed sets in the links. Suppose now that we also have

(i) another fixed set $\left(\mathcal{C}^{\prime}, \partial \mathcal{C}^{\prime}\right)$ in $\overline{\mathcal{M}} \backslash \mathcal{R}$ bounding another collection $F^{\prime}$ of 2 -dimensional $\pi^{\prime}$-fixed sets in the Taubes boundary $X$, and

(ii) the intersection $\mathcal{C} \cap \mathcal{C}^{\prime}$ contains a singular stratum with larger isotropy group $\pi^{\prime \prime} \neq \pi^{\prime}$. 
In that case, following the outline of the argument given in [12, the next step is to pick a subgroup $C_{p} \subseteq \pi^{\prime}$, for some prime $p$, and perturb into $C_{p}$-general position. The difficulty is that the union $\mathcal{C} \cup \mathcal{C}^{\prime}$ could now be perturbed into a smooth $C_{p}$-fixed cobordism between the two collections of 2-spheres, which is allowable.

However, if this "bad" case occurs, the existence of such a stratum $\mathcal{C}^{\prime}$ would imply that the sum of the two-dimensional homology classes in $F^{\prime}$ would map to zero under the Taubes map $\tau_{*}: H_{2}(X ; \mathbf{Z}) \rightarrow H_{2}\left(\mathcal{M}^{*} ; \mathbf{Z}\right)$. Furthermore, $\mu\left(\left[F^{\prime}\right]\right)=0 \in H^{2}\left(\mathcal{B}^{*} ; \mathbf{Z}\right)$ by [5, 5.1.2]. But the composite

$$
H_{2}(X ; \mathbf{Z}) \stackrel{\mu}{\longrightarrow} H^{2}\left(\mathcal{B}^{*} ; \mathbf{Z}\right) \stackrel{\tau^{*}}{\longrightarrow} H^{2}(X ; \mathbf{Z})
$$

is just the Poincaré duality map [5, 5.3.3], so $\tau^{*}\left(\mu\left(\left[F^{\prime}\right]\right)\right)=0$ implies that $\left[F^{\prime}\right]=0 \in H_{2}(X ; \mathbf{Z})$. An easy modification of [12, Corollary 4] shows that, whenever $\operatorname{Fix}\left(H_{2}(X ; \mathbf{Z} / p), C_{p}\right) \neq 0$, then the 2-dimensional components of $\operatorname{Fix}\left(X, C_{p}\right)$ represent linearly independent elements of $H_{2}(X ; \mathbf{Z} / p)$. It follows that $\tau_{*}\left(\left[F^{\prime}\right]\right) \neq 0$ in $H_{2}\left(\mathcal{M}^{*} ; \mathbf{Z}\right)$, and hence no such stratum $\mathcal{C}^{\prime}$ bounding $F^{\prime}$ exists.

Therefore, after the perturbation into $C_{p}$-general position, the perturbed bounding set $\mathcal{C}$ will be a smooth $C_{p}$-fixed null-bordism for $F$ in $\mathcal{M}^{*}$, disjoint from the Taubes boundary. The existence of such a null-bordism is now ruled out by the $\mu-$ map argument exactly as given in [12, Lemma 12].

The following useful statements were proved in the argument just given.

\section{Corollary 2.15 [12, Theorem 16]}

(i) No non-empty collection of 2-dimensional $\pi^{\prime}$-fixed sets in the links of reducible connections bounds a compact fixed set in $\mathcal{M}^{*}$.

(ii) If $\operatorname{Fix}\left(\mathcal{R}, \pi^{\prime}\right) \neq \emptyset$, then no non-empty collection $F$ of 2 -dimensional $\pi^{\prime}$ fixed sets in $X$, with $[F] \neq 0$, bounds a compact fixed set in $\mathcal{M}^{*}$.

(iii) If there exists a $\pi^{\prime}$-fixed 2 -sphere in $X$, representing a non-zero homology class, then $\operatorname{Fix}\left(\mathcal{R}, \pi^{\prime}\right) \neq \emptyset$.

Remark 2.16 The case $X=S^{4}(1,0)$ shows that the first assumption in part (ii) is necessary. In general, if $\operatorname{Fix}\left(\mathcal{R}, \pi^{\prime}\right)=\emptyset$ then the trace of the action of a generator of $\pi^{\prime}$ on $H_{2}(X ; \mathbf{Z})$ is zero, and so $\chi\left(\operatorname{Fix}\left(X, \pi^{\prime}\right)=2\right.$. It follows that $\operatorname{Fix}\left(X, \pi^{\prime}\right)$ consists of two isolated points, or a single null-homologous 2-sphere, which must bound a compact fixed set in $\mathcal{M}^{*}$. We will see in the next section that the assumption $[F] \neq 0$ in part (ii) is not actually necessary. 
Definition 2.17 Let $[D]$ be a $\pi^{\prime}$-fixed reducible connection, with $\pi^{\prime} \neq 1$. A $\pi^{\prime}$-incident stratum at $[D]$ is a connected component $\mathcal{C}$ of $\mathcal{M}_{\left(\pi^{\prime}\right)}^{*}$ such that $[D] \in \overline{\mathcal{C}}$. The stabilizer $\pi_{\mathcal{C}}$ of $\mathcal{C}$ is the subgroup of $\pi$ leaving the stratum invariant.

A $\pi^{\prime}$-incident stratum intersected with the cone $\mathcal{N}_{D}$ of the $\pi^{\prime}$-linear action at a reducible $[D]$ is a connected component of $\left(\mathcal{N}_{D} \backslash[D]\right)_{\left(\pi^{\prime}\right)}$. Since its closure has $[D]$ as a limit point, $\pi^{\prime} \subseteq \pi_{D} \subseteq \pi_{\mathcal{C}}$.

Remark 2.18 There are at most three proper $\pi^{\prime}$-incident strata at a reducible connection since $\left(\ell(D), \pi_{D}\right)$ is a linear action on $\mathbb{C} P^{2}$. Their closures consist of either (i) three 1-dimensional $\pi^{\prime}$-fixed strata intersecting $\ell(D)$ in three isolated $\pi^{\prime}$-fixed points, or (ii) a 1 -dimensional and a 3 -dimensional $\pi^{\prime}$-fixed stratum intersecting $\ell(D)$ in an isolated $\pi^{\prime}$-fixed point and a $\pi^{\prime}$-fixed 2 -sphere, respectively. It follows that the Euler characteristic $\chi\left(\operatorname{Fix}\left(\ell(D), \pi^{\prime}\right)\right)=3$ for each reducible $[D] \in \mathcal{R}$, and each $\pi^{\prime} \subseteq \pi_{D}$.

Proposition 2.19 Let $\mathcal{C}$ be a $\pi^{\prime}$-incident stratum with stabilizer $\pi_{\mathcal{C}}$. Then there exists a $\pi_{\mathcal{C}}$-invariant neighbourhood $\nu(\overline{\mathcal{C}})$ of $\overline{\mathcal{C}} \cap \mathcal{M}^{*}$, such that $g(\nu(\overline{\mathcal{C}})) \cap$ $\nu(\overline{\mathcal{C}})=\emptyset$ for all $g \in \pi, g \notin \pi_{\mathcal{C}}$. In addition, the disjoint union $\pi \times_{\pi_{\mathcal{C}}} \nu(\overline{\mathcal{C}})$ is a smooth $\pi$-invariant submanifold of $\mathcal{M}^{*}$ on which the moduli space is equivariantly transverse. The closure $\overline{\mathcal{C}}$ is one of the following types:

(i) $\overline{\mathcal{C}} \cap \partial \overline{\mathcal{M}}=\emptyset$, $\operatorname{dim} \mathcal{C}=1$, and $\mathcal{C}$ has exactly two reducible limit points,

(ii) $\overline{\mathcal{C}} \cap \partial \overline{\mathcal{M}} \neq \emptyset$, $\operatorname{dim} \mathcal{C}=1$ or 3 , and $\mathcal{C}$ contains a unique reducible limit point, or

(iii) $\overline{\mathcal{C}} \cap \partial \overline{\mathcal{M}} \neq \emptyset, \operatorname{dim} \mathcal{C}=3$ and $\mathcal{C}$ has more than one reducible limit point.

Proof We begin with the classification of incident strata into types. First we consider case (i) when $\overline{\mathcal{C}} \cap \partial \overline{\mathcal{M}}=\emptyset$ and $\operatorname{dim} \mathcal{C}=1$. If $\pi^{\prime}=\pi_{D}=\pi$, then such a 1 -dimensional smooth stratum has no interior limiting endpoint in $\mathcal{M}^{*}$ by general position. In that case, its other limiting endpoint must be another reducible $\left[D_{j}\right]$ (distinct from its initial limit reducible $\left[D_{i}\right]$ by Corollary 2.8 ).

If $\pi^{\prime}=\pi_{D} \neq \pi$, and if $\mathcal{C}$ has an interior limiting endpoint $[A] \in \mathcal{M}^{*}$, then $\pi^{\prime} \subsetneq$ $\pi_{A}$ and the orbit $\pi_{A} \times_{\pi_{D}} \mathcal{C}$ is a $\pi_{A}$-invariant collection of 1 -dimensional strata which are incident with the orbit $\pi \times_{\pi_{D}}[D]$ and have $[A]$ as a common limit point. However, this possibility is eliminated by Corollary 2.8(ii). It follows that our incident 1 -dimensional stratum $\mathcal{C}$ has no interior limiting endpoint in $\mathcal{M}^{*}$. Since we also assumed that its closure didn't meet $\partial \overline{\mathcal{M}}$, the only 
remaining possibility is that its other limiting endpoint must be a reducible $\left[D_{j}\right] \neq\left[D_{i}\right]$. That completes case (i).

Next we note that the possibility $\overline{\mathcal{C}} \cap \partial \overline{\mathcal{M}}=\emptyset$ and $\operatorname{dim} \mathcal{C}=3$ is ruled out by Lemma 2.14 In the remaining cases (ii) and (iii) we suppose that $\overline{\mathcal{C}} \cap \partial \overline{\mathcal{M}} \neq \emptyset$. When $\mathcal{C}$ has a unique reducible limit point, both $\operatorname{dim} \mathcal{C}=1$ and $\operatorname{dim} \mathcal{C}=3$ both occur in the linear models. On the other hand, if $\mathcal{C}$ has more than one reducible limit point, and some other limit point in $X$, then it can't be 1-dimensional, since a 1 -manifold has at most two ends. It follows in case (iii) that $\operatorname{dim} \mathcal{C}=3$.

Now that we have the classification of incident strata into types, we can observe that in each case $\mathcal{C}$ is incident with a part of the moduli space (either $\mathcal{N}_{D}$ or $\mathcal{M}_{\lambda_{0}}$ ) where the $\mathcal{M}^{*}$ is cut out equivariantly transversely. But then Lemma 2.9 implies the existence of a $\pi_{\mathcal{C}}$-invariant neighbourhood $\nu(\overline{\mathcal{C}})$ with the required properties.

Corollary 2.20 The closures of distinct $\pi^{\prime}$-incident strata can intersect only at reducibles.

Proof For each limit reducible $[D]$, the group $\pi_{D}$ operates freely, away from the "zero section" $\overline{\mathcal{C}}$, within each of the $\pi_{D}$-invariant neighbourhoods $\nu(\overline{\mathcal{C}}) \subset$ $\mathcal{M}^{*}$. It follows that $\mathcal{C}$ is the unique $\pi^{\prime}$-fixed stratum in $\nu(\overline{\mathcal{C}})$.

\section{The structure of the singular set}

The main result of this section is Corollary 3.9. showing that there is a nice subset of $\operatorname{Fix}\left(\overline{\mathcal{M}}, \pi^{\prime}\right)$ containing $\operatorname{Fix}\left(\mathcal{R}, \pi^{\prime}\right)$ and $\operatorname{Fix}\left(X, \pi^{\prime}\right)$ which is path connected for each $\pi^{\prime} \subseteq \pi$. From this we can deduce the proof of Theorem B and prepare for the proof of Theorem A in the next section.

\section{(3.A) Connecting the fixed-point sets}

In the last section we studied the individual $\pi^{\prime}$-incident strata at the reducible connections. Let $\mathcal{C}\left(\pi^{\prime}\right)$ denote the union of the $\pi^{\prime}$-incident strata at all $[D] \in$ $\operatorname{Fix}\left(\mathcal{R}, \pi^{\prime}\right)$, assuming that $\operatorname{Fix}\left(\mathcal{R}, \pi^{\prime}\right) \neq \emptyset$. Notice that the $\pi$-action permuting the reducibles induces a $\pi$-action on $\mathcal{C}\left(\pi^{\prime}\right)$ which permutes the type (i) strata. Each type (ii) or (iii) stratum $\mathcal{C}$ has a stabilizer subgroup $\pi_{\mathcal{C}}$ containing $\pi^{\prime}$.

For $\pi^{\prime} \neq 1$, we now consider the following union of $\pi^{\prime}$-fixed sets:

$$
\mathcal{F}\left(\pi^{\prime}\right):=\operatorname{Fix}\left(\mathcal{R}, \pi^{\prime}\right) \cup \operatorname{Fix}\left(X, \pi^{\prime}\right) \cup \bigcup\left\{\overline{\mathcal{C}}\left(\pi^{\prime \prime}\right) \mid \pi^{\prime} \subseteq \pi^{\prime \prime}\right\}
$$


lying in the compactified moduli space $(\overline{\mathcal{M}}, \pi)$. By Corollary 2.20 this is a disjoint union except for inclusion of strata, and possible common limit points among the $\pi^{\prime}$-fixed reducibles. The main result of this section is that $\mathcal{F}\left(\pi^{\prime}\right)$ is connected.

Remark 3.1 If $\operatorname{Fix}\left(\mathcal{R}, \pi^{\prime}\right)=\emptyset$, then we define $\mathcal{F}\left(\pi^{\prime}\right)$ as the connected component of $\operatorname{Fix}\left(\overline{\mathcal{M}}^{*}, \pi^{\prime}\right)$ containing $\operatorname{Fix}\left(X, \pi^{\prime}\right)$. This exists since $\operatorname{Fix}\left(X, \pi^{\prime}\right)$ consists either of two isolated points or a null-homologous 2 -sphere, and either case these bound in $\overline{\mathcal{M}}^{*}$.

If we remove from $\mathcal{F}\left(\pi^{\prime}\right)$ the open cones around each of the reducibles in $\operatorname{Fix}\left(\mathcal{R}, \pi^{\prime}\right)$, we obtain a disjoint union of smooth $\pi^{\prime}$-fixed cobordisms between the singular sets in the links $\left\{\ell(D) \mid[D] \in \operatorname{Fix}\left(\mathcal{R}, \pi^{\prime}\right)\right\}$ and the singular set $\operatorname{Fix}\left(X, \pi^{\prime}\right)$ (see Proposition 2.19). Note that all the components of $\operatorname{Fix}\left(X, \pi^{\prime}\right)$ are connected to the reducibles by $\pi^{\prime}$-incident strata (Corollary 2.13 and Proposition 2.15). We wish to compare the total Euler characteristic of the stratum cobordisms at the two ends.

Let $\chi\left(\operatorname{Fix}\left(X, \pi^{\prime}\right)\right)=r+2$, where $r$ is the number of $\pi^{\prime}$-fixed reducibles, and let $\ell$ denote the number of homologically zero 2 -sphere components in $\operatorname{Fix}\left(X, \pi^{\prime}\right)$. We will assign weight $\chi(\mathcal{C})=2$ to each type (i) stratum, and weight $\chi(\mathcal{C})=$ $2(k-1)$ to each type (iii) stratum with $k$ reducible limit points. The type (ii) strata have weight zero. The quantity

$$
\chi:=\sum\left\{\chi(\mathcal{C}) \mid \mathcal{C} \in \mathcal{F}\left(\pi^{\prime}\right)\right\}
$$

is the total Euler characteristic used up in joining various reducibles by closures of $\pi^{\prime}$-incident strata. Next we let $\tau(\mathcal{C})=2(t-1)$, where $t$ denotes the number of 2-sphere boundary components in $\operatorname{Fix}\left(X, \pi^{\prime}\right)$ in a type (ii) or type (iii) stratum $\mathcal{C}$ (otherwise let $\tau(\mathcal{C})=0$ ), and define the excess

$$
\tau:=\sum\left\{\tau(\mathcal{C}) \mid \mathcal{C} \in \mathcal{F}\left(\pi^{\prime}\right)\right\}
$$

We have the basic relation

$$
3 r-\chi+\tau+2 \ell=r+2
$$

by comparing Euler characteristics, but there is actually enough information to compute these quantities:

Lemma 3.2 Let $1 \neq \pi^{\prime} \subseteq \pi$. If $\operatorname{Fix}\left(\mathcal{R}, \pi^{\prime}\right) \neq \emptyset$, then the weight $\chi=2(r-1)$ and the excess $\tau=0$. In addition, there are no homologically trivial 2-sphere components in $\operatorname{Fix}\left(X, \pi^{\prime}\right)$. 
Proof Each linear action on $\mathbb{C} P^{2}$ contributes Euler characteristic 3 to the $\pi^{\prime}$-incident strata, so we have an "excess" of $3 r-\chi$ which must equal the contribution from $\operatorname{Fix}\left(X, \pi^{\prime}\right)$. We can divide the $r$ reducibles in $\operatorname{Fix}\left(\mathcal{R}, \pi^{\prime}\right)$ into $s$ disjoint subsets by defining two reducibles to be equivalent if they are both limit points of the same stratum $\mathcal{C}$. Suppose there are $k_{i}$ reducibles in the $i^{t h}$ subset, for $1 \leq i \leq s$, and $\sum k_{i}=r$. Each subset accounts for $2\left(k_{i}-1\right)$ units in $\chi$. Then the equation

$$
\chi=\sum 2\left(k_{i}-1\right)=2 r-2 s=2(r-1)+\tau+2 \ell
$$

gives $2(1-s)=\tau+2 \ell \geq 0$. Since $s \geq 1$ we are done.

Corollary 3.3 Suppose that $\operatorname{Fix}(\mathcal{R}, \pi)=\emptyset$, and $\pi^{\prime} \neq 1$ is a proper subgroup which is maximal with respect to the property $\operatorname{Fix}\left(\mathcal{R}, \pi^{\prime}\right) \neq \emptyset$. Then there exists a unique $\pi^{\prime}$-incident stratum of type (iii), and a unique 2-sphere component in $\operatorname{Fix}\left(X, \pi^{\prime}\right)$.

Proof Suppose if possible that there are no $\pi^{\prime}$-incident strata of type (iii). Then the set $\mathcal{F}\left(\pi^{\prime}\right)$ contains $r$ reducibles connected in pairs by $(r-1)$ type (i) strata. But $\pi$ acts on $\mathcal{F}\left(\pi^{\prime}\right)$ by permuting the reducibles, and since $\pi$ has odd order, there would exist a $\pi$-fixed reducible, contrary to our assumption. Now if $\mathcal{C}$ is a $\pi^{\prime}$-incident stratum of type (iii), it must intersect $X$ in a $\pi^{\prime}$-fixed 2 -sphere $F$. If $\pi_{\mathcal{C}} \neq \pi$, then the orbit $\pi \times_{\pi_{\mathcal{C}}} \mathcal{C}$ would contribute $2\left|\pi / \pi_{\mathcal{C}}\right|$ to the Euler characteristic of $\operatorname{Fix}\left(X, \pi_{\mathcal{C}}\right)$, contrary to our calculation of $\chi$. Therefore $\pi_{\mathcal{C}}=\pi$ and $F$ is $\pi$-invariant.

Since $\operatorname{Fix}(\mathcal{R}, \pi)=\emptyset$, the fixed set $\operatorname{Fix}(X, \pi)$ consists of two isolated points $x_{0}, x_{1} \in F$. Now suppose that there is another 2 -sphere component $F^{\prime}$ in $\operatorname{Fix}\left(X, \pi^{\prime}\right)$. By Proposition 2.15 it is contained in a $\pi^{\prime}$-incident type (ii) or (iii) stratum $\mathcal{C}^{\prime}$. We conclude by counting as above, that $\pi_{\mathcal{C}^{\prime}}=\pi$, and it follows that $F \cap F^{\prime}=\left\{x_{0}, x_{1}\right\}$. But this contradicts the effectiveness of the $\pi$-action at $x_{0}$ (by consideration of the tangential isotropy representation $T_{x_{0}} X$ ).

Corollary 3.4 If $\operatorname{Fix}(\mathcal{R}, \pi)=\emptyset$, there are at most two maximal, proper subgroups $\pi_{1}, \pi_{2}$, such that $\operatorname{Fix}\left(\mathcal{R}, \pi_{i}\right) \neq \emptyset$. If two such subgroups exist, then $\pi_{1} \cap \pi_{2}=\{1\}$.

Proof Suppose that $\pi_{1}$ and $\pi_{2}$ are maximal proper subgroups with the given property. By the last result, they each have a fixed 2-sphere $F_{1}, F_{2}$ respectively, and $F_{1} \cap F_{2}$ gives just the two isolated points $\left\{x_{0}, x_{1}\right\}$ in $\operatorname{Fix}(X, \pi)$. In order to have an effective action at $x_{0}$, we must have $\pi_{1} \cap \pi_{2}=\{1\}$ and no other such subgroup can exist in the linear model for $\left(\mathbb{R}^{4}, C_{m}\right)$. 
For a type (iii) stratum $\mathcal{C}$, suppose that $\mathcal{R}(\mathcal{C})=\left\{\left[D_{1}\right], \ldots,\left[D_{k}\right]\right\}$ is the set of its reducible limit points. The stabilizer group $\pi_{\mathcal{C}}$ of $\mathcal{C}$ acts by permuting the reducibles in $\mathcal{R}(\mathcal{C})$. We identify the following sub-types:

Lemma 3.5 Suppose that $1 \neq \pi^{\prime} \subset \pi$. For each $\pi^{\prime}$-incident type (iii) stratum, the set $\mathcal{R}(\mathcal{C})$ of reducible limit points, under the permutation action of the stratum stabilizer group, is either

(iii-a) fixed by $\pi_{\mathcal{C}}$, so $\pi_{D}=\pi_{\mathcal{C}}$ for all $[D] \in \mathcal{R}(\mathcal{C})$, and $\pi^{\prime}=\pi_{\mathcal{C}}$, or

(iii-b) a disjoint union of free $\pi_{\mathcal{C}} / \pi^{\prime}$-orbits, with $\pi^{\prime}=\pi_{D}$ for all $[D] \in \mathcal{R}(\mathcal{C})$, and $\pi^{\prime} \subsetneq \pi_{\mathcal{C}}$, or

(iii-c) a disjoint union of free $\pi_{\mathcal{C}} / \pi^{\prime}$-orbits, together with some $\pi_{\mathcal{C}}$-fixed points $[D]$, and $\pi^{\prime} \subsetneq \pi_{D}=\pi_{\mathcal{C}}$.

Proof Each $\pi^{\prime}$-incident type (iii) stratum $\overline{\mathcal{C}}$ has a 2 -sphere boundary component in $\operatorname{Fix}\left(X, \pi^{\prime}\right)$ on which the stabilizer subgroup $\pi_{\mathcal{C}}$ either acts trivially,or semi-freely with two isolated $\pi_{\mathcal{C}}$-fixed points. In the first case, $\pi_{\mathcal{C}}$ also acts trivially on the stratum $\mathcal{C}$ and fixes all the reducibles in $\mathcal{R}(\mathcal{C})$. In the second case, $\pi^{\prime} \subsetneq \pi_{\mathcal{C}}$, and the two isolated $\pi_{\mathcal{C}}$-fixed points either lie in $\operatorname{Fix}(X, \pi)$ and bound a 1 -dimensional $\pi$-fixed set in $\mathcal{M}^{*}$, or lie in distinct 1 -dimensional type (ii) strata (for $\pi_{\mathcal{C}}$ ) which both have $\pi_{\mathcal{C}}$-fixed limit reducibles $[D]$. In that case, these limit reducibles are among the set of $\pi_{\mathcal{C}}$-fixed point in $\mathcal{R}(\mathcal{C})$. The remaining limit reducibles are permuted freely by $\pi_{\mathcal{C}} / \pi^{\prime}$.

Corollary 3.6 Suppose that $\operatorname{Fix}(\mathcal{R}, \pi)=\emptyset$, and $\operatorname{Fix}\left(\mathcal{R}, \pi^{\prime}\right) \neq \emptyset$ for some $\pi^{\prime} \neq 1$. Let $\pi^{\prime \prime} \supseteq \pi^{\prime}$ be a maximal subgroup such that $\operatorname{Fix}\left(\mathcal{R}, \pi^{\prime \prime}\right) \neq \emptyset$. If $\mathcal{C}_{0}$ is the unique $\pi^{\prime \prime}$-incident stratum of type (iii), then $\pi_{\mathcal{C}_{0}}=\pi$, and the set of limit reducibles $\mathcal{R}\left(\mathcal{C}_{0}\right)$ is a disjoint union of free $\pi / \pi^{\prime \prime}$-orbits.

This stratum $\mathcal{C}_{0}$ will be called the maximal type (iii-b) stratum for $\pi^{\prime}$. It is uniquely determined by any subgroup $\pi^{\prime} \subseteq \pi^{\prime \prime}$ such that $\operatorname{Fix}\left(\mathcal{R}, \pi^{\prime}\right) \neq \emptyset$.

\section{(3.B) The singular set tree}

In order to show that the sets $\mathcal{F}\left(\pi^{\prime}\right)$ are connected, we will study the configuration of reducibles and incident strata more abstractly.

Definition 3.7 For each subgroup $1 \neq \pi^{\prime} \subseteq \pi$, we will associate a graph

$$
\Gamma\left(\pi^{\prime}\right):=\Gamma(V, E)
$$


whose vertex set $V:=\operatorname{Fix}\left(\mathcal{R}, \pi^{\prime}\right)$, provided that $\operatorname{Fix}(\mathcal{R}, \pi) \neq \emptyset$. If $\operatorname{Fix}(\mathcal{R}, \pi)=$ $\emptyset$, we adjoin one more $\pi$-fixed vertex $v_{0}$, called the root vertex, which is common to all the graphs $\Gamma\left(\pi^{\prime}\right), \pi^{\prime} \subseteq \pi$.

The edge set $E$ is determined by the classification of the $\pi^{\prime}$-incident strata in $\mathcal{F}\left(\pi^{\prime}\right)$ into types (see Proposition 2.19). For a $\pi^{\prime \prime}$-incident stratum of type (iii-a) with $\pi^{\prime} \subseteq \pi^{\prime \prime}=\pi_{\mathcal{C}}$, we pick an ordering $\left\{\left[D_{1}\right], \ldots,\left[D_{k}\right]\right\}$ of the limit reducibles, and extend the ordering $\pi$-equivariantly over the orbit $\pi \times_{\pi_{\mathcal{C}}} \mathcal{R}(\mathcal{C})$. For a $\pi^{\prime \prime}$-incident stratum $\mathcal{C}$ of type (iii-c) we pick a $\pi_{\mathcal{C}}$-fixed reducible $[D]$, called the branch vertex, and extend the choice by $\pi$-equivariance over the orbit of $\mathcal{C}$.

Two distinct vertices $\left[D_{i}\right],\left[D_{j}\right]$ are joined by an edge if and only if:

(e-1) there is a $\pi^{\prime \prime}$-incident stratum of type (i), with $\pi^{\prime} \subseteq \pi^{\prime \prime}$, and limit reducibles $\left[D_{i}\right]$ and $\left[D_{j}\right]$, or

(e-2) there is a $\pi^{\prime \prime}$-incident stratum $\mathcal{C}$ of type (iii-a), with $\pi^{\prime} \subseteq \pi^{\prime \prime}=\pi_{\mathcal{C}}$, limit reducibles $\left[D_{i}\right],\left[D_{j}\right]$, and $j=i+1$ in the chosen ordering of $\mathcal{R}(\mathcal{C})$, or

(e-3) there is a $\pi^{\prime \prime}$-incident stratum $\mathcal{C}$ of type (iii-c), with $\pi^{\prime} \subseteq \pi^{\prime \prime} \subsetneq \pi_{\mathcal{C}}$, limit reducibles $\left[D_{i}\right],\left[D_{j}\right]$, such that $\left[D_{i}\right]$ is the branch vertex in $\mathcal{C}$ and $\pi_{D_{j}}=\pi^{\prime \prime}$.

If $\operatorname{Fix}(\mathcal{R}, \pi)=\emptyset$, the root vertex $v_{0}$ is joined to a vertex $[D]$ if and only if $[D]$ is a limit reducible in the maximal type (iii-b) stratum $\mathcal{C}_{0}$ for $\pi^{\prime}$.

Recall that a connected graph with no circuits is called a tree.

Corollary 3.8 For each subgroup $1 \neq \pi^{\prime} \subseteq \pi$, the graph $\Gamma\left(\pi^{\prime}\right)$ is a tree.

Proof The graph $\Gamma\left(\pi^{\prime}\right)$ contains no closed circuits, by Corollary 2.8, so we must show that $\Gamma\left(\pi^{\prime}\right)$ is connected. Let's first consider the case where $\operatorname{Fix}(\mathcal{R}, \pi)$ $\neq \emptyset$. We have the basic relation $\chi=2(r-1)$ where $r$ is the number of $\pi^{\prime}-$ fixed reducibles. In our Euler characteristic count, each edge $e \in E$ arising from a type (i) stratum contributes $\chi(e)=2$, and each type (iii) stratum with $k$ reducible limit points contributes $\chi(e)=2(k-1)$, so we can interpret the quantity $\chi$ as twice the number of edges $e \in E$. In other words, there are exactly $(r-1)$ edges in $\Gamma\left(\pi^{\prime}\right)$. But a tree with $k$ vertices has exactly $(k-1)$ edges, so if $\Gamma\left(\pi^{\prime}\right)$ were disconnected into $\ell$ trees of $k_{i}$ vertices, $1 \leq k_{i} \leq \ell$, we would get

$$
\chi=\sum 2\left(k_{i}-1\right)=2 r-2 \ell \geq 2(r-1) .
$$

Therefore $\ell=1$ and $\Gamma\left(\pi^{\prime}\right)$ is connected. 
If $\operatorname{Fix}(\mathcal{R}, \pi)=\emptyset$, but $\operatorname{Fix}\left(\mathcal{R}, \pi^{\prime}\right) \neq \emptyset$, let $\pi^{\prime \prime}$ be a maximal subgroup with this property. Then the $k$ limit reducibles of the maximal $\pi^{\prime}$-fixed stratum $\mathcal{C}_{0}$ of type (iii-b) are permuted in a disjoint union of $\pi / \pi^{\prime \prime}$-orbits. This stratum adds $k$ edges to the graph $\Gamma\left(\pi^{\prime}\right)$, instead of $(k-1)$, and the total number of edges is given by $\frac{1}{2}(\chi+2)=r$ since $\mathcal{C}$ is incident with a $\pi^{\prime}$-fixed 2 -sphere in $X$. It follows by counting as before that $\Gamma\left(\pi^{\prime}\right)$ is connected.

Corollary 3.9 For each $\pi^{\prime} \subseteq \pi$, the set $\mathcal{F}\left(\pi^{\prime}\right)$ is path connected.

Proof We may assume that $\operatorname{Fix}\left(\mathcal{R}, \pi^{\prime}\right) \neq \emptyset$. By construction of the graph $\Gamma\left(\pi^{\prime}\right)$, and Corollary 2.20, it is clear that $\Gamma(V, E)$ is connected if $\mathcal{F}\left(\pi^{\prime}\right)$ is connected. On the other hand, all the components of $\operatorname{Fix}\left(X, \pi^{\prime}\right)$ are connected to the reducibles by $\pi^{\prime}$-incident strata. Therefore if $\Gamma\left(\pi^{\prime}\right)$ is connected then $\mathcal{F}\left(\pi^{\prime}\right)$ is connected.

The internal structure of the tree $\Gamma\left(\pi^{\prime}\right)$ is clarified by considering the stabilizer $\pi_{\mathcal{F}}$ of a subset $\mathcal{F}\left(\pi^{\prime}\right)$. We will use the well-known fact that a finite group of odd order acting on a tree always fixes a vertex.

Lemma 3.10 For each set $\mathcal{F}\left(\pi^{\prime}\right), 1 \neq \pi^{\prime} \subseteq \pi$, there exists $[D] \in \operatorname{Fix}\left(\mathcal{R}, \pi^{\prime}\right)$ such that $\pi_{D}=\pi_{\mathcal{F}}$, and $\pi_{D}$ is a maximal element in the set of subgroups $\left\{\pi_{D^{\prime}}\right\}$ for all $\left[D^{\prime}\right] \in \operatorname{Fix}\left(\mathcal{R}, \pi^{\prime}\right)$. Moreover, $\mathcal{F}\left(\pi_{D}\right)=\operatorname{Fix}\left(\mathcal{F}\left(\pi^{\prime}\right), \pi_{D}\right)$.

Proof We note first that $\pi_{D^{\prime}} \subseteq \pi_{\mathcal{F}}$ for all $\left[D^{\prime}\right] \in \operatorname{Fix}\left(\mathcal{R}, \pi^{\prime}\right)$ because $\mathcal{F}\left(\pi^{\prime}\right)$ is connected. On the other hand, the $\pi_{\mathcal{F}}$-action on the tree $\Gamma\left(\pi^{\prime}\right)$ has a fixed vertex $[D] \in \operatorname{Fix}\left(\mathcal{R}, \pi^{\prime}\right)$, so $\pi_{\mathcal{F}} \subseteq \pi^{\prime}$. Therefore $\pi_{\mathcal{F}}=\pi_{D}$ for this reducible. Since $\pi^{\prime} \subseteq \pi_{\mathcal{F}}=\pi_{D}$, we get the obvious inclusion $\mathcal{F}\left(\pi_{D}\right) \subseteq \operatorname{Fix}\left(\mathcal{F}\left(\pi^{\prime}\right), \pi_{D}\right)$. But if $\mathcal{C}$ is a $\pi^{\prime \prime}$-incident stratum at a $\pi_{D}$-fixed reducible, for $\pi^{\prime} \subseteq \pi^{\prime \prime}$, then either it is 1-dimensional and fixed by $\pi_{D}$ as well, or it is 3-dimensional and the $\pi_{D}$-fixed set of its closure is in $\mathcal{F}\left(\pi_{D}\right)$.

We now want to consider the full singular set of $(\mathcal{M}, \pi)$, or at least those components whose closures intersect either the links of the reducibles or the Taubes collar. We define

$$
\mathcal{F}(X, \pi):=\bigcup\left\{\mathcal{F}\left(\pi^{\prime}\right) \mid 1 \neq \pi^{\prime} \subseteq \pi\right\}
$$

and define the associated graph

$$
\Gamma(X, \pi):=\bigcup\left\{\Gamma\left(\pi^{\prime}\right) \mid 1 \neq \pi^{\prime} \subseteq \pi\right\}
$$

The main result is: 
Theorem 3.11 The graph $\Gamma(X, \pi)$ is a tree and the singular set $\mathcal{F}(X, \pi)$ is connected.

Proof Suppose that $[D]$ and $\left[D^{\prime}\right]$ are reducible connections. If $\pi_{D} \subseteq \pi_{D^{\prime}}$ then $\left[D^{\prime}\right] \in \mathcal{F}\left(\pi_{D}\right)$ so we can connect them by a path in $\mathcal{F}\left(\pi_{D}\right)$, or equivalently by an edge path in $\Gamma\left(\pi_{D}\right)$. If $\operatorname{Fix}(\mathcal{R}, \pi) \neq \emptyset$, this shows that any connection $[D]$ can be connected to any $\pi$-fixed reducible $\left[D^{\prime}\right]$, and we are done. If $\operatorname{Fix}(\mathcal{R}, \pi)=\emptyset$ but $\pi_{D} \neq 1$ for some reducible connection $[D]$, then we let $\mathcal{C}_{0}$ denote the maximal type (iii) stratum for $\pi_{D}$. By Lemma 3.5 the stabilizer $\pi^{\prime}=\pi_{D^{\prime}}$ for any reducible $\left[D^{\prime}\right] \in \mathcal{R}\left(\mathcal{C}_{0}\right)$ is the maximal subgroup containing $\pi_{D}$ with respect to the property that $\operatorname{Fix}\left(\mathcal{R}, \pi^{\prime}\right) \neq \emptyset$. Then $\left[D^{\prime}\right] \in \mathcal{F}\left(\pi_{D}\right)$ and $\left[D^{\prime}\right]$ is connected to the root vertex in $\Gamma\left(\pi_{D}\right)$. Alternately, $\left[D^{\prime}\right]$ is connected to $\operatorname{Fix}(X, \pi)=\left\{x_{0}, x_{1}\right\}$ inside $\overline{\mathcal{C}}_{0}$. Therefore $\Gamma(X, \pi)$ and $\mathcal{F}(X, \pi)$ are connected, and $\Gamma(X, \pi)$ is a tree.

\section{(3.C) The proof of Theorem B}

After this preparation we can now show that a smooth pseudo-free action of an odd order cyclic group on $X \simeq \#_{1}^{n} \mathbb{C} P^{2}$ must be semi-free. This answers a question of A Edmonds [7, who pointed out that the hypothesis of a smooth action is necessary. Edmonds showed [7, Theorem 5.4] that $C_{25}$ can act locally linearly and pseudo-freely on $X=\#_{1}^{n} \mathbb{C} P^{2}$, for $n=10$, inducing the representation $\mathbf{Z}\left[C_{5}\right] \oplus \mathbf{Z}\left[C_{5}\right]$ on $H_{2}(X ; \mathbf{Z})$. In particular, by computing traces, we see that this action is not semi-free.

We begin with the following observation.

Lemma 3.12 Let $(X, \pi)$ be a pseudo-free smooth action of $\pi=C_{m}, m$ odd, on $X \simeq \#_{1}^{n} \mathbb{C} P^{2}$. Then there are only type (i) or 1-dimensional type (ii) strata in the moduli space $\left(\mathcal{M}^{*}, \pi\right)$.

Proof We have already established in Proposition 2.19 that the $\pi^{\prime}$-incident strata come in three types, and those of type (ii) or (iii) intersect $\operatorname{Fix}\left(X, \pi^{\prime}\right)$ in isolated points or 2-spheres. By Proposition 2.15 and Corollary 3.3, any $\pi^{\prime}$-fixed 2-sphere in $\mathcal{N}_{D}$ for a reducible $[D]$ would imply the existence of a singular 2 -sphere in $(X, \pi)$. Since the given action is pseudo-free, this can't occur.

The usefulness of this observation is shown by the following two remarks. 
Lemma 3.13 Suppose that $\mathcal{C}$ is a $\pi^{\prime}$-incident stratum of type (i), with limit reducibles $[D]$ and $\left[D^{\prime}\right]$. Then $\pi_{D}=\pi_{D^{\prime}}$.

Proof Since $\mathcal{C}$ is 1 -dimensional, its intersection with $\mathcal{N}_{D}$ is fixed by $\pi_{D}$, hence its intersection with $\mathcal{N}_{D^{\prime}}$ is also fixed by $\pi_{D}$. Therefore $\pi_{D} \subseteq \pi_{D^{\prime}}$. Similarly, $\pi_{D^{\prime}} \subseteq \pi_{D}$

Corollary $3.14 \pi_{D}=\pi_{D^{\prime}}$ for all $[D],\left[D^{\prime}\right] \in \mathcal{R}$ with non-trivial stabilizer.

We now finish the proof of Theorem B by using again the well-known fact that a finite group of odd order acting on a tree always fixes a vertex. In our case, the group $\pi=C_{m}$ of odd order acts on the tree $\Gamma(X, \pi)$, so there is a vertex $[D] \in \mathcal{R}$ which is fixed by $\pi$. But this means that $\pi \subseteq \pi_{D}$ so $\pi_{D}=\pi$ for all $[D] \in \mathcal{R}$ with non-trivial stabilizer. In addition, we could have some free $\pi$-orbits of reducibles, but these don't contribute to the singular set.

It follows that every type (ii) stratum is also fixed by $\pi$, so the limit points of these strata in $X$ consist entirely of isolated $\pi$-fixed points. On the other hand, by Corollary 2.13 any $\pi^{\prime}$-singular point in $X$ is a limit point of some type (ii) stratum. Therefore the singular set of $(X, \pi)$ consists of isolated $\pi$-fixed points, and the action is semi-free.

\section{Stratified cobordisms and the proof of Theorem A}

In this section we will show that the moduli space contains an equivariant connected sum $(X(\mathbb{T}), \pi)$ of linear actions on $\mathbb{C} P^{2}$, and provides an equivariant stratified cobordism between $(X(\mathbb{T}), \pi)$ and our given action $(X, \pi)$. By construction, the action $(X(\mathbb{T}), \pi)$ will have the same permutation representation on $H_{2}(X ; \mathbf{Z})$ as the given action. The stratified cobordism will have smooth strata, and equivariant vector bundle neighbourhoods. This will allow us to compare the isotropy groups and rotation numbers in $(X, \pi)$ with those of $(X(\mathbb{T}), \pi)$.

We begin by describing how to realize edges in $\Gamma(X, \pi)$ by thickened paths in $\overline{\mathcal{M}}^{*}$. Recall that the tree $\Gamma(X, \pi)$ may have some edges arising from type (iii) incident strata, listed as cases (e-2) and (e-3) in Definition 3.7. If $\mathcal{C}$ is a $\pi^{\prime}$ incident stratum of type (iii-c) with $\pi^{\prime} \subsetneq \pi_{\mathcal{C}}$, then there exists a branch vertex $[D] \in \mathcal{R}(\mathcal{C})$ and each of the limit reducibles appearing in free $\pi_{\mathcal{C}} / \pi^{\prime}$-orbits are joined by an edge to the branch vertex. We realize this geometrically by choosing a smooth embedded path $\gamma$ in $\overline{\mathcal{C}}$ from $[D]$ to some other reducible 
$\left[D^{\prime}\right] \in \mathcal{R}(\mathcal{C})_{\left(\pi^{\prime}\right)}$. The interior of this path will be chosen to lie in $\mathcal{M}_{\left(\pi^{\prime}\right)}^{*}$, with the property that $g \cdot \gamma$ is disjoint from $\gamma$ (except at $[D]$ ) for all $1 \neq g \in \pi_{\mathcal{C}}$. Now let $\nu_{1}(\mathcal{C})$ denote the union of small tubular neighbourhoods in $\mathcal{M}^{*}$. around the paths $\left\{g \cdot \gamma \mid g \in \pi_{\mathcal{C}}\right\}$. We extend by equivariance to the orbit $\pi \times_{\pi_{\mathcal{C}}} \nu_{1}(\mathcal{C})$.

For a type (iii-a) $\pi^{\prime}$-incident stratum $\mathcal{C}$ with $\pi^{\prime}=\pi_{\mathcal{C}}$ we have chosen an ordering

$$
\left\{\left[D_{1}\right], \ldots,\left[D_{k}\right]\right\}
$$

of the limit reducibles. We choose disjoint smooth embedded paths $\gamma_{i}$ in $\overline{\mathcal{C}}$, from $\left[D_{i}\right]$ to $\left[D_{i+1}\right]$, for $1 \leq i \leq k-1$, and thicken as before to define $\nu_{1}(\mathcal{C})$. We again extend by equivariance to the orbit $\pi \times_{\pi_{\mathcal{C}}} \nu_{1}(\mathcal{C})$.

If $\operatorname{Fix}(\mathcal{R}, \pi)=\emptyset$ we will also have at most two maximal type (iii-b) strata, associated to subgroups $\pi_{1}, \pi_{2}$ with $\pi_{1} \cap \pi_{2}=\{1\}$, which are maximal with respect to the property $\operatorname{Fix}\left(\mathcal{C}, \pi^{\prime}\right) \neq \emptyset$. In this case, we have $\operatorname{Fix}(X, \pi)=$ $\left\{x_{0}, x_{1}\right\}$, so we can define $\nu\left(x_{0}\right.$ to be a small $\pi$-invariant 5 -ball in the Taubes collar centered at the fixed point $\left(x_{0}, \lambda_{0} / 2\right)$. The boundary of this 5 -ball is a linear action $S_{0}:=S^{4}(a, b)$ having invariant 2 -spheres with isotropy $\pi_{1}$ and $\pi_{2}$. Now if $\mathcal{C}_{0}$ is a maximal type (iii-b) stratum for $\pi_{1}$, its limit reducibles are a disjoint union of free $\pi / \pi_{1}$-orbits. We choose disjoint smooth paths $\gamma_{i}, \pi-$ equivariantly, from a disjoint union of free $\pi / \pi_{1}$-orbits of points in $\operatorname{Fix}\left(S_{0}, \pi_{1}\right)$ to the limit reducibles in $\mathcal{C}_{0}$. Then we thicken these paths as above, and adjoin $\nu\left(x_{0}\right)$ to define $\nu_{1}\left(\mathcal{C}_{0}\right)$.

Finally, there may exist some free $\pi$-orbits of reducibles. If $\pi \times[D]$ is such an orbit, let $\nu_{1}(D)$ denote the union of a $\pi$-invariant collection of disjoint, thickened, smooth paths from $\{g \cdot[D]: g \in \pi\}$ to a free $\pi$-orbit of points in some $\partial \mathcal{N}_{D^{\prime}}$, if there exists a $\pi$-fixed reducible $\left[D^{\prime}\right]$, or in $S_{0}$ if $\operatorname{Fix}(\mathcal{R}, \pi)=\emptyset$.

Definition 4.1 We define a subset of $\overline{\mathcal{M}}^{*}$ containing all the reducible connections. Let

$$
\mathcal{N}(X, \pi):=\bigcup\left\{\mathcal{N}_{D}:[D] \in \mathcal{R}\right\}
$$

and

$$
\nu(X, \pi):=\bigcup\{\nu(\mathcal{C}): \mathcal{C} \text { type }(\mathrm{i}) \text { stratum }\} \cup\left\{\nu_{1}(\mathcal{C}): \mathcal{C} \text { type (iii) stratum }\right\},
$$

and then define

$$
\mathcal{D}(X, \pi):=\mathcal{N}(X, \pi) \cup \nu(X, \pi) \cup \bigcup\left\{\nu_{1}(D): \pi_{D}=\{1\}\right\}
$$

Theorem 4.2 The boundary $(Y, \pi):=\partial \mathcal{D}(X, \pi)$ is an equivariant connected sum of linear actions on $\mathbb{C} P^{2}$, and $H_{2}(Y ; \mathbf{Z}) \cong H_{2}(X ; \mathbf{Z})$ as permutation modules. 
Proof The equivariant moduli space $(\mathcal{M}, \pi)$ has given us an admissible, weighted tree $\mathbb{T}$ based on $\Gamma(X, \pi)$. The weights are given by the linear actions on $\mathbb{C} P^{2}$ in the links $\ell(D)$ of the reducible connections, and by construction the permutation action on $H_{2}(X ; \mathbf{Z})$ is realized by the permutation action on the reducibles, which are all contained in $\mathcal{D}(X, \pi)$. The edges of $\mathbb{T}$ are given by the strata $\mathcal{C}$ of type (i) together with the paths $\gamma$ constructed in the definition of the subsets $\nu_{1}(\mathcal{C})$ for the type (iii) strata.

The proof of Theorem A Let $W:=W(X, \pi)$ denote the complement of the interior of $\mathcal{D}(X, \pi)$ in $\left(\overline{\mathcal{M}}^{*}, \pi\right)$. Then $W$ is a stratifed $\pi$-equivariant cobordism between $(X, \pi)$ and the equivariant connected sum $(Y, \pi)$. In addition, the tubular neigbourhoods of the singular strata in $W$ are just the intersections $W \cap \nu(\mathcal{C})$ for all type (ii) or (iii) strata in $\mathcal{M}^{*}$, together with a thickened tube from $D\left(x_{0}\right)$ in $X$ to $S_{0}$ in the case $\operatorname{Fix}(\mathcal{R}, \pi)=\emptyset$. It follows that the isotropy structure of $(Y, \pi)$ is the same as that of $(X, \pi)$. In addition, these tubular neighbourhoods are the total spaces of equivariant vector bundles over the strata in $W$, so the rotation numbers at singular points in $(Y, \pi)$ match up with rotation numbers in $(X, \pi)$.

The proof of Theorem $\mathbf{C}$ This follows from Theorem A and Theorem 1.15. Combining Theorem A with Theorem 1.16 gives the stable realization theorem for permutation modules in the case when $\operatorname{Fix}(\mathcal{R}, \pi)=\emptyset$, or equivalently, in the case when there are no trivial summands $\mathbf{Z}$ in $H_{2}(X ; \mathbf{Z})$.

\section{References}

[1] Edward Bierstone, General position of equivariant maps, Trans. Amer. Math. Soc. 234 (1977) 447-466

[2] Glen E Bredon, Introduction to compact transformation groups, Pure and Applied Mathematics, Vol. 46, Academic Press, New York (1972)

[3] S K Donaldson, An application of gauge theory to four-dimensional topology, J. Differential Geom. 18 (1983) 279-315

[4] S K Donaldson, The orientation of Yang-Mills moduli spaces and 4-manifold topology, J. Differential Geom. 26 (1987) 397-428

[5] S K Donaldson, P B Kronheimer, The geometry of four-manifolds, Oxford Mathematical Monographs, Oxford Science Publications, Clarendon Press and Oxford University Press, New York (1990)

[6] Allan L Edmonds, Aspects of group actions on four-manifolds, Topology Appl. 31 (1989) 109-124 
[7] Allan L Edmonds, Periodic maps of composite order on positive definite 4manifolds, preprint (2000) 20 pages

[8] Ronald Fintushel, Terry Lawson, Compactness of moduli spaces for orbifold instantons, Topology Appl. 23 (1986) 305-312

[9] Ronald Fintushel Ronald J Stern, Pseudofree orbifolds, Ann. of Math. 122 (1985) 335-364

[10] Daniel S Freed, Karen K Uhlenbeck, Instantons and four-manifolds, second ed., Mathematical Sciences Research Institute Publications, vol. 1, SpringerVerlag, New York (1991

[11] Ian Hambleton, Ronnie Lee, Perturbation of equivariant moduli spaces, Math. Ann. 293 (1992) 17-37

[12] Ian Hambleton, Ronnie Lee, Smooth group actions on definite 4-manifolds and moduli spaces, Duke Math. J. 78 (1995) 715-732

[13] Sören Illman, Every proper smooth action of a Lie group is equivalent to a real analytic action: a contribution to Hilbert's fifth problem, from: "Prospects in topology (Princeton, NJ, 1994)", Ann. of Math. Stud. vol. 138, Princeton Univ. Press, Princeton, NJ (1995) 189-220

[14] John N Mather, Stratifications and mappings, from: "Dynamical systems (Proc. Sympos., Univ. Bahia, Salvador, 1971)", Academic Press, New York (1973) 195-232

[15] J P Serre, Trees, Graduate Texts in Mathematics, Springer-Verlag, New York (1980)

[16] Mihail Tanase, Smooth finite cyclic group actions on positive definite 4manifolds, $\mathrm{PhD}$ thesis, McMaster University (2003) viii+112 pages 